\title{
Kalman filtering with faded measurements
}

\author{
Subhrakanti Dey*, Alex S. Leong, Jamie S. Evans \\ ARC Special Research Centre for Ultra-Broadband Information Networks (CUBIN), Department of Electrical and Electronic Engineering, University of Melbourne, \\ Parkville, Vic. 3010, Australia
}

\section{A R T I C L E I N F O}

Article history:

Received 5 October 2008

Received in revised form

10 June 2009

Accepted 27 June 2009

Available online 31 July 2009

Keywords:

Fading channels

Kalman filtering

Sensor networks

Stability

\begin{abstract}
A B S T R A C T
This paper considers a sensor network where single or multiple sensors amplify and forward their measurements of a common linear dynamical system (analog uncoded transmission) to a remote fusion center via noisy fading wireless channels. We show that the expected error covariance (with respect to the fading process) of the time-varying Kalman filter is bounded and converges to a steady state value, based on some earlier results on asymptotic stability of Kalman filters with random parameters. More importantly, we provide explicit expressions for sequences which can be used as upper bounds on the expected error covariance, for specific instances of fading distributions and scalar measurements (per sensor). Numerical results illustrate the effectiveness of these bounds.
\end{abstract}

(C) 2009 Elsevier Ltd. All rights reserved.

\section{Introduction}

Due to a recent steady growth of activity in wireless sensor networks with a large number of nodes monitoring an environment/ object in various applications, multi-sensor based estimation of random processes under limited resources and communication constraints has led to new challenging filtering problems. In particular, estimation of dynamical systems based on observations received from multiple sensors under these constraints is known to be a potentially hard problem, especially in the case of data networks connecting the sensors to the remote fusion center (FC), where the estimation algorithm is implemented. Delay and/or communication outage (or measurement loss) in such networks can give rise to instability in the average estimation error at the FC. Instead of using a digital data network between a sensor and the FC, an alternative approach is to use uncoded transmission where each sensor simply amplifies and forwards its measurement to the FC using analog communication. It was shown in Gastpar and Vetterli (2003) that, for a Gaussian sensor network, where multiple sensors observe a Gaussian source, such an amplify and forward protocol

\footnotetext{
is This work was supported by the Australian Research Council. The material in this paper was partially presented at 2008 Allerton Conference on Communication, Control and Computing, Monticello, IL, USA, September 24-26, 2008. This paper was recommended for publication in revised form by Associate Editor George Yin under the direction of Editor Ian R. Petersen.

* Corresponding author. Tel.: +613 83446299; fax: +61383446678.

E-mail addresses: sdey@unimelb.edu.au (S. Dey), asleong@unimelb.edu.au (A.S. Leong), jse@unimelb.edu.au (J.S. Evans).
}

combined with a perfectly synchronized simultaneous transmission from the sensors (resulting in a coherent addition of transmitted signals at the FC) leads to an asymptotically optimal estimation (based on minimum mean square estimation) at the $\mathrm{FC}$, in that the estimation error at the FC decays as $1 / M$ for a sufficiently large $M$ where $M$ is the number of sensors, as opposed to digital transmission (with separate source and channel coding) where the estimation error decays as $1 / \log M$. Furthermore, an uncoded analog transmission system is simple to implement and results in little delay. Motivated by these results, we focus on a similar amplify and forward strategy based sensor network estimating a linear dynamical system. In this scenario, the sensors simply amplify and forward their measurements of the linear system to a remote FC via randomly time-varying fading channels, where they are received in noise. The optimal state estimation filter at the fusion center (with perfect knowledge of the fading channel gains at the FC) is still a (time-varying) Kalman filter where the error covariance is given by a Riccati difference equation, albeit with random fading parameters. Studying the asymptotic stability properties of this random parameter Riccati equation in terms of the average (over the fading process) error covariance is the main purpose of this paper. In addition, we provide concrete results on how to obtain deterministic and asymptotically convergent bounding sequences on the average error covariances which are hard to compute analytically.

The topic of stability analysis of time-varying and random parameter Riccati equations is not new. Early results on detectability and stabilizability of time-varying systems include Anderson and Moore (1981) and Jazwinski (1970), though strong uniformity 
conditions which are difficult to verify have to be satisfied. Less restrictive conditions are considered in Bougerol $(1993,1995)$, and asymptotic properties studied. The results obtained include the almost sure exponential stability of the Kalman filter, and the existence of a unique stationary distribution for the error covariance. A suboptimal linear filter using only channel statistics is derived and analyzed in Rajasekaran, Satyanarayana, and Srinath (1971) and Tugnait (1981) for the so-called linear systems with "multiplicative noise" (the system model in this paper is similar to this). However, for unstable systems, the error covariance of this estimator becomes unbounded. Studies of the stability of the Kalman filter via expectation of the error covariance was done in Sinopoli et al. (2004) for measurement losses (over a single communication link) undergoing a Bernoulli process, and showed the existence of a threshold such that, if the measurement arrival rate lies below this threshold, then the expected error covariance becomes unbounded. These results were extended in various directions: to Markovian measurement loss processes in Huang and Dey (2007) and Xie and Xie (2008), to a network of communication links in Dana, Gupta, Hespanha, Hassibi, and Murray (2007), to communication links with controlled transmission schemes in $\mathrm{Xu}$ and Hespanha (2005), and to multiple sensors sharing a multi-access channel in Zhu, Sinopoli, Poolla, and Sastry (2007). In all these papers, the results focus on finding the threshold (or bounds thereof) on the measurement arrival probability, below which the expected error covariance becomes unbounded. For continuous fading distributions, an analysis of the average error covariance for scalar systems and Rayleigh fading can be found in Mostofi and Murray (2005), where it is found that the expected error covariance is always bounded. Stability of random Riccati equations was also studied in Yuan and Guo (1999) under a certain stochastic observability condition, and some boundedness conditions on the random matrices in the system model.

In this paper, we study a linear system (in particular for unstable systems), the noisy observations (via single or multiple sensors) of which are being sent over independent and identically distributed block-fading channels to a remote FC. Using some rather general asymptotic stability results for linear systems with ergodic parameters from Bougerol (1995), we show that, under some mild conditions, the expected (with respect to the fading process) error covariance matrix of the Kalman filter remains bounded and converges to a steady state matrix from arbitrary positive semidefinite matrix initial conditions. This result is in contrast with the recent results in Sinopoli et al. (2004) which show that the expected error covariance matrix for unstable systems in a situation where measurements can be lost with a non-zero probability can become unbounded if this loss probability exceeds a certain threshold. While this observation may not be surprising from the results and discussions in Bougerol (1995), Mostofi and Murray (2005) and Yuan and Guo (1999), we believe that this observation needs to be made in a general sense. In addition, for special cases of vector state and scalar measurements, and scalar state and measurements (for both single sensor and multiple sensor scenarios), and specific fading distributions, we provide explicit bounding matrix (or scalar) sequences that overbounds the expected error covariance matrix and also converges to a steady state value. These bounds provide a simple way to compute realistic (and often quite tight) bounds on the expected error covariance, and can be quite useful in situations when one wants to minimize the expected error covariance for such sensor network based estimation problems to optimally allocate resources across multiple sensors. When an exact recursive expression for the average error covariance is not available, one can minimize its upper bound instead, for which we provide exact recursive formulas. Problems of this nature can be solved by dynamic programming techniques and will be addressed elsewhere.

The rest of the paper is organized as follows. Section 2 presents the various signal models treated in this paper and states the assumptions under which we prove our results. Section 3 presents the convergence results for the various signal models and the results on the bounding sequences, along with numerical illustrations. Finally, Section 4 concludes the paper with some discussion on future research. Proofs are relegated to the Appendix unless otherwise stated.

\section{System model}

We consider a discrete-time linear time invariant system that represents a phenomenon of interest (for example, the trajectory of a moving object) given by

$x_{k+1}=A x_{k}+w_{k}$

where $x_{k} \in \mathbb{R}^{n}, w_{k} \in \mathbb{R}^{n}, A \in \mathbb{R}^{n \times n}$. We assume that $\left\{w_{k}\right\}$ is white ${ }^{1}$ and follows a Gaussian distribution with zero mean and variance $\Sigma_{w}>0$. Note here that a matrix $V>0$ implies that $V$ is a positive definite matrix. Similarly, $V \geq 0$ implies $V$ is a positive semidefinite matrix. We also assume that the initial distribution of $x_{0}$ is Gaussian with mean zero and covariance matrix $P_{0} \geq 0$. Note that, in this work, we allow $A$ to be an unstable matrix. Indeed, the results presented in this paper are interesting only when $A$ is unstable, as in Sinopoli et al. (2004).

This system is observed by a sensor or a number of sensors which yield discrete-time measurements of the state of the system. These measurements are then sent over a wireless medium to a central processing unit called the Fusion Centre (FC). We assume that the sensors use analog forwarding as in Gastpar and Vetterli (2003) to send the measurements to the FC, i.e, they simply amplify and forward their measurements to the FC. Due to the randomly time-varying nature of the wireless medium, the FC then receives faded versions of all the measurements in additive noise either separately (orthogonal access) or as a sum of all received measurements in noise (non-orthogonal access). We consider the single sensor and multiple sensor cases separately.

\subsection{Single sensor case}

In this case, the linear time invariant system (1) is observed by a single sensor which produces a discrete-time measurement $y_{k}$ which is given by

$y_{k}=C x_{k}+v_{k}$

where $y_{k} \in \mathbb{R}^{m}, v_{k} \in \mathbb{R}^{m}, C \in \mathbb{R}^{m \times n}$. We assume that $\left\{v_{k}\right\}$ is white and Gaussian distributed with zero mean and variance $\Sigma_{v}>0$. Denoting the $i$ th element of the measurement and measurement noise vectors as $y_{k}^{i}$ and $v_{k}^{i}$ respectively where $i=1,2, \ldots, m$, we assume that the sensor transmitter amplifies the component $y_{k}^{i}$ by a factor $\alpha_{k}^{i}$ and sends it to the FC over a fading channel with channel gain $h_{k, i}$. We assume that the channel undergoes slow fading such that the phase of the complex channel can be estimated and compensated for at the receiver, so that essentially $h_{k, i}$ represents the real-valued envelope of the complex channel gain. We also assume that the channel gain remains constant over the time interval to send the $i$ th component, $i=1,2, \ldots, m$ but one can have $h_{k, i} \neq h_{k, j}$ for $i \neq j$ and $i, j \in\{1,2, \ldots, m\}$. This assumption is valid when each measurement interval is much larger than the coherence time of the fading channel, which is likely to be the case in low bandwidth sensor network applications. Denoting $h_{k}=\left(h_{k, 1} h_{k, 2} \ldots h_{k, m}\right)$, we also assume that $h_{k}$ is independently and identically distributed according to a continuous fading distribution $f(h)$ such that $P\left(h_{k, i}>0\right)=1$, $\forall k, i$.

\footnotetext{
1 We say that a discrete-time process $\left\{w_{k}\right\}$ is white if $w_{k}$ and $w_{l}$ are independent
} for $k \neq l$. 
The FC receives a scaled version of each component of the measurement vector added with measurement noise, in additive noise at the FC, which represents the channel noise in the communication channel between the sensor and the FC. We assume that all the measurement components are sent separately to the FC via orthogonal channels within the measurement time interval. The received signal vector at the FC then can be written as

$z_{k}=H_{k} C x_{k}+H_{k} v_{k}+n_{k}$

where $H_{k}=\operatorname{diag}\left(\alpha_{k}^{1} h_{k, 1} \quad \alpha_{k}^{2} h_{k, 2} \ldots \alpha_{k}^{m} h_{k, m}\right)$ and $n_{k}=\left(\begin{array}{ll}n_{k}^{1} & n_{k}^{2}\end{array}\right.$ $\left.\ldots n_{k}^{m}\right)^{\prime}$ represents the channel noise vector. For simplicity, we assume that $\left\{n_{k}^{i}\right\},\left\{n_{k}^{j}\right\}$ are mutually independent for $i \neq j$ and $n_{k}^{i}$ is Gaussian distributed with zero mean and variance $\sigma_{n_{i}}^{2}, \forall k$. Thus, $n_{k}$ is identically Gaussian distributed with zero mean and variance $\Sigma_{n}=\operatorname{diag}\left(\sigma_{n_{1}}^{2} \sigma_{n_{2}}^{2} \ldots \sigma_{n_{m}}^{2}\right)$. We also assume that $\left\{n_{k}\right\}$ is white and $x_{0},\left\{h_{k}\right\},\left\{v_{k}\right\},\left\{w_{k}\right\}$ and $\left\{n_{k}\right\}$ are mutually independent.

For simplicity we will also assume that $\alpha_{k}^{i}=1$ for all $i=$ $1,2, \ldots, m$ and for all $k=1,2, \ldots$. Note that, when the state space model (1) is stable, $\alpha_{k}^{i}$ is usually chosen to satisfy the power constraint at the sensor transmitter. However, for the unstable case, this choice will make $\alpha_{k}^{i} \rightarrow 0$ as $k \rightarrow \infty$. We therefore choose $\alpha_{k}^{i}=1, \forall i, k$ although this will require the sensor transmitter to transmit with exponentially increasing power. This is justified by the fact that the expected error covariance matrix converges to a bounded matrix (as seen later through numerical simulations) reasonably fast, thus making the results derived in this paper meaningful, even within a finite time horizon during which the sensor transmitter power will remain bounded. In Bougerol (1995), it was shown that the error covariance matrix, starting from an arbitrary positive semidefinite matrix as the initial condition, converges to a stationary process exponentially fast (see also Lemma 3.2). However, an analytic computation of the rate of convergence for these results will hinge on a Lyapunov exponent analysis of products of random matrices and is beyond the scope of this paper.

With the assumption that $\alpha_{k}^{i}=1, \forall i, k$, we now have $H_{k}=$ $\operatorname{diag}\left(h_{k, 1} h_{k, 2} \ldots h_{k, m}\right)$. The overall state-space model for this system can now be written as

$x_{k+1}=A x_{k}+w_{k}, \quad z_{k}=H_{k} C x_{k}+\bar{v}_{k}$

where $\bar{v}_{k}=H_{k} v_{k}+n_{k}$. Since $H_{k}$ is a diagonal matrix, it is easy to see that $\bar{v}_{k}$ is Gaussian distributed with zero mean and time-varying covariance matrix $R_{k}=H_{k} \Sigma_{v} H_{k}+\Sigma_{n}$.

Assumption 2.1. We make the standard assumption that the pair $\left(A, \Sigma_{w}^{\frac{1}{2}}\right)$ is stabilizable and the pair $(A, C)$ is detectable.

For technical reasons, in order to use some results from Bougerol (1995) later, ${ }^{2}$ we will also make the assumption:

Assumption 2.2. (i) $A$ is invertible.

(ii) $\max \left(0, \log \left\|H_{0} C\right\|\right)$ is integrable.

(iii) $\left\{H_{k}\right\}$ is stationary and ergodic.

For discrete time systems which are obtained by discretizing a continuous time system, Assumption 2.2(i) will be satisfied (see e.g. Chan, Goodwin, \& Sin, 1984). Assumption 2.2(ii) is also satisfied by commonly used fading distributions such as Rayleigh

\footnotetext{
2 The model (4) does not quite correspond to the model of Bougerol (1995), in that $\Sigma_{w}$ is not the identity matrix and $R_{k}$ is time-varying. But if we let $\breve{x}_{k}=$ $\Sigma_{w}^{-1 / 2} x_{k}, \breve{A}=\Sigma_{w}^{-1 / 2} A \Sigma_{w}^{1 / 2}, \breve{w}_{k}=\Sigma_{w}^{-1 / 2} w_{k}, \breve{C}_{k}=\left(H_{k} \Sigma_{v} H_{k}+\Sigma_{n}\right)^{-1 / 2} H_{k} C \Sigma_{w}^{1 / 2}$, $\breve{v}_{k}=\left(H_{k} \Sigma_{v} H_{k}+\Sigma_{n}\right)^{-1 / 2}\left(H_{k} v_{k}+n_{k}\right), \breve{z}_{k}=\left(H_{k} \Sigma_{v} H_{k}+\Sigma_{n}\right)^{-1 / 2} z_{k}$, we then end up with the model $\breve{x}_{k+1}=\breve{A} \breve{x}_{k}+\breve{w}_{k}, \breve{z}_{k}=\breve{C}_{k} \breve{x}_{k}+\breve{v}_{k}$, with $\breve{w}_{k}$ and $\breve{v}_{k}$ both having unit covariance.
}

or Nakagami. Assumption 2.2(iii) is automatically satisfied since we assumed $\left\{H_{k}\right\}$ to be i.i.d., though the results of Bougerol (1995) can also hold in more general cases where the channel has memory.

In what follows, we will also consider several special cases of the above general model (4). In particular, we will consider the following scalar state/scalar measurement model:

$x_{k+1}=a x_{k}+w_{k}, \quad z_{k}=h_{k} x_{k}+h_{k} v_{k}+n_{k}$

where $x_{k}, z_{k}, w_{k}, v_{k}, n_{k}$ are all scalar random processes (with $w_{k} \sim$ $\left.N\left(0, \sigma_{w}^{2}\right), v_{k} \sim N\left(0, \sigma_{v}^{2}\right), n_{k} \sim N\left(0, \sigma_{n}^{2}\right)\right)$. We have taken $c=1$ without loss of any generality, as both sides can be scaled by $c$ to obtain the model described above. Just as before, we assume that $\left\{h_{k}\right\}$ is a sequence of i.i.d random variables distributed according to a continuous fading distribution $f(h)$ such that $P\left(h_{k}>0\right)=1, \forall k$.

In addition, we will consider a vector state/scalar measurement model for the single-sensor case given by

$x_{k+1}=A x_{k}+w_{k}, \quad z_{k}=h_{k} \bar{c} x_{k}+h_{k} v_{k}+n_{k}$

where $\bar{c} \in \mathbb{R}^{1 \times n}$ is given by $\bar{c}=\left(\bar{c}_{1}, \bar{c}_{2}, \ldots, \bar{c}_{n}\right)$ and $v_{k}, n_{k}$ are scalars, where $x_{k}, w_{k}$ follow the same model as in (4), and $n_{k},\left\{h_{k}\right\}$ are described by the same models as in (5).

\subsection{Multisensor case}

In the multisensor case, we assume that the dynamical process (1) is observed by $M$ sensors each producing a scalar measurement. While the general results on convergence and bounds derived in the next section for the single sensor case can be extended to the multisensor case, for simplicity, we stick to a scalar state and scalar measurement (per sensor) model. The sensors can then communicate their measurements to the FC via either a multi-access channel (Gastpar \& Vetterli, 2003) (where all sensors transmit simultaneously without the time/frequency division multiplexing) or via orthogonal channels (Cui, Xiao, Goldsmith, Luo, \& Poor, 2007).

In the multi-access scheme, we assume that the phase shift in each channel is compensated by distributed transmit beamforming, so that the measurements from all sensors add up coherently at the FC. Note that although this may be difficult to implement in practice, especially for large sensor networks, it can still be achieved by the distributed synchronization scheme described in Mudumbai, Barriac, and Madhow (2007). Additionally, in studies such as Li and Dai (2007) it has been shown, in slightly different contexts, that, for moderate amounts of phase error, much of the potential performance gains can still be retained. Mathematically, the signal model for the multisensor multi-access scheme is given by

$x_{k+1}=a x_{k}+w_{k}, \quad z_{k}=\sum_{i=1}^{M} h_{k, i}\left(c_{i} x_{k}+v_{k}^{i}\right)+n_{k}$.

We assume that $v_{k}^{i} \sim N\left(0, \sigma_{v_{i}}^{2}\right), n_{k} \sim N\left(0, \sigma_{n}^{2}\right)$ and $\left\{v_{k}^{i}\right\},\left\{v_{k}^{j}\right\}$ are mutually independent for $i \neq j, i, j \in\{1,2, \ldots, M\}$. Similarly, $\left\{h_{k, i}\right\},\left\{h_{k, j}\right\}$ are statistically independent for $i \neq$ $j, i, j \in\{1,2, \ldots, M\}$. Note that $h_{k, i}$ may not be identically distributed for all $i$. Due to the distributed transmit beamforming assumption, note that $h_{k, i}, \forall i$ denotes the non-negative channel fading amplitude. We also assume here that $c_{i}>0$ for all sensors without loss of generality. Note that if $c_{l}$ for sensor $l$ is negative, one can choose the amplification factor for this sensor as -1 instead of 1 . This assumption is there to ensure that the distributed transmit beamforming scheme works effectively.

In the orthogonal access scheme, the FC simply receives a vector consisting of the individual faded measurements from all the sensors. We can write the signal model as

$x_{k+1}=a x_{k}+w_{k}, \quad z_{k}^{i}=h_{k, i} c_{i} x_{k}+h_{k, i} v_{k}^{i}+n_{k}^{i}$ 
for $i=1, \ldots, M$, where $z_{k}^{i}$ denotes the received signal (at the FC) from the $i$ th sensor and $n_{k}^{i}$ is the channel noise for the $i$ th sensor's channel. In this case, the FC observation consists of the vector $\left(z_{k}^{1}, z_{k}^{2}, \ldots, z_{k}^{M}\right)^{\prime}$ and the other modelling assumptions regarding $\left\{h_{k, i}\right\}, v_{k}^{i}$ remain the same as in (7). $n_{k}^{i}$ is independently and identically distributed with $N\left(0, \sigma_{n_{i}}^{2}\right)$ and $\left\{n_{k}^{i}\right\},\left\{n_{k}^{j}\right\}$ are mutually independent for $i \neq j$. The mutual independence assumption amongst $x_{0}$ and the various noise processes remains the same as before. Note that unlike (7) however, there is no need to assume $c_{i}>0, \forall i$ in this case.

\section{Convergence results and bounds on the expected error covariance matrix}

In this section, we present some convergence and boundedness results on the average (over the channel fading distribution) error covariance matrix for the optimal one step ahead predictor for the vector state vector measurement system (4). Later, we will specialize these results for the various cases given by (5), (6) for the single sensor case and (7) and (8) for the multisensor case, for specific fading distributions. Using the knowledge of these distributions and inequalities involving some special functions, we derive more specific bounds for these cases.

We assume that the FC has full knowledge of the system matrices and noise covariances, including the time-varying channel fading matrices $H_{k}$. The above state-space model (4) is a linear time-varying system and the optimal predictor (filter) for this system is a time-varying Kalman predictor (filter), that can be constructed at the FC. Denote $\mathcal{Z}_{k}=\left(z_{0}, z_{1}, \ldots, z_{k}\right)$ and $\mathscr{H}_{k}=\left(H_{0}\right.$, $\left.H_{1}, \ldots, H_{k}\right)$, and define the one step ahead optimal predictor and its error covariance as

$\hat{x}_{k+1 \mid k}=E\left[x_{k+1} \mid \mathcal{Z}_{k}, \mathscr{H}_{k}\right]$

$P_{k+1 \mid k}=E\left[\left(x_{k+1}-\hat{x}_{k+1 \mid k}\right)\left(x_{k+1}-\hat{x}_{k+1 \mid k}\right)^{\prime} \mid \mathcal{Z}_{k}, \mathscr{H}_{k}\right]$

where / denotes the transpose operation. In the following, we will use the shorthand notation $P_{k+1}$ for $P_{k+1 \mid k}$. Using the time-varying Kalman filtering equations, one can easily derive that the prediction error covariance matrix $P_{k}$ satisfies the following discrete-time time-varying Riccati equation:

$P_{k+1}=A\left[P_{k}-P_{k} C^{\prime} H_{k}\left(H_{k} C P_{k} C^{\prime} H_{k}+R_{k}\right)^{-1} H_{k} C P_{k}\right] A^{\prime}+\Sigma_{w}$

with $R_{k}=H_{k} \Sigma_{v} H_{k}+\Sigma_{n}$. It is straightforward to show that the above equation can be rewritten as

$P_{k+1}=A\left[P_{k}-P_{k} C^{\prime}\left(L_{k}^{-1}+C P_{k} C^{\prime}+\Sigma_{v}\right)^{-1} C P_{k}\right] A^{\prime}+\Sigma_{w}$

where $L_{k}=H_{k} \Sigma_{n}^{-1} H_{k}$. It can be easily shown that, starting with any $P_{0} \geq 0$, one retains the positive semidefinite nature for all $P_{k}$, see e.g. Anderson and Moore (1979).

Remark 1. Note that here we make the assumption that the fading parameters $H_{k}$ are perfectly known at the FC. This is a fairly common assumption in the wireless communication community, also known as channel state information at the receiver (CSIR). For a slow fading channel, as assumed here, channel estimation can be carried out at the FC by periodically sending powerful pilot signals to the sensors which allow the sensors to compute the channel gains and send them as overhead information to the FC. Note also that, here, the channel from the FC to each sensor is assumed to be identical to that from the sensor to the FC. This is known as channel reciprocity, and holds for systems where information is communicated between the transmitter and receiver using the same frequency but at different time slots (known as time division duplex (TDD) protocol).
It should be obvious that $E_{\mathcal{H}_{k}}\left[P_{k+1}\right]=E_{\mathscr{H}_{k-1}}\left[G\left(P_{k}\right)\right]$ where the expectation is taken with respect to the channel realization history $\mathscr{H}_{k}=\left\{H_{1}, H_{2}, \ldots, H_{k}\right\}$, and

$$
\begin{aligned}
G\left(P_{k}\right) & =E_{H_{k}}\left[A\left[P_{k}-P_{k} C^{\prime}\left(L_{k}^{-1}+C P_{k} C^{\prime}+\Sigma_{v}\right)^{-1} C P_{k}\right] A^{\prime}+\Sigma_{w} \mid P_{k}\right] \\
& =A\left[P_{k}-P_{k} C^{\prime} E_{H_{k}}\left[\left(L_{k}^{-1}+C P_{k} C^{\prime}+\Sigma_{v}\right)^{-1}\right] C P_{k}\right] A^{\prime}+\Sigma_{w}
\end{aligned}
$$

where the last line in the above equation follows due to the fact that $P_{k}$ is adapted to $\mathscr{H}_{k-1}$ and $\left\{H_{k}\right\}$ is a sequence of i.i.d random matrices. Below, for notational simplicity, we will drop the subscript from the expectation operator whenever the random process over which the expectation is taken is obvious from the context. Define the space of $n \times n$ positive semidefinite matrices as $\wp_{n}$. Then we have the following property for $G: \wp_{n} \rightarrow \wp_{n}$,

Lemma 3.1. The matrix-valued function $G(X)$ is a concave nondecreasing function of $X \in \varsigma_{n}$.

See Appendix A for the proof.

Now, given $(A, C)$ is a detectable pair, it is easy to show that so is $\left(A, H_{k} C\right)$ for $H_{k}$ invertible, i.e. $h_{k, i}>0, \forall i$. Then $\left(A, H_{k} C\right)$ is an almost surely weakly detectable pair (for the definition of almost sure weak detectability, see definition 2.4 of Bougerol, 1995). Thus, under Assumptions 2.1 and 2.2, all the conditions of Theorem 5.6 of Bougerol (1995) are satisfied. It then follows that the Kalman filter (the error covariance of which is defined by the time-varying Riccati equation (10)) is almost surely exponentially stable. This essentially implies that (starting from any $P_{0} \in \gamma_{n}$ ), if the gain matrix associated with $(10)$ is denoted as $\bar{K}_{k}=$ $A P_{k} C^{\prime} H_{k}\left(H_{k} C P_{k} C^{\prime} H_{k}+R_{k}\right)^{-1}$, then the sequence $\left\{\bar{M}_{k}=A-\right.$ $\left.\bar{K}_{k} H_{k} C, k \in \mathbb{N}\right\}$ is almost surely exponentially stable. This definition of almost sure exponential stability for a sequence of random matrices defined on a probability space $(\Omega, \mathcal{F}, \mathcal{P})$ is given by the following property (see definition 1.1 of Bougerol, 1995): for any $\epsilon>0$ and almost all $\omega \in \Omega$, there exists $\gamma>0$ and $J(\omega)>0$ such that

$\left\|\bar{M}_{k-1}(\omega) \bar{M}_{k-2}(\omega) \ldots \bar{M}_{k-n}(\omega)\right\| \leq J(\omega) \mathrm{e}^{-n \gamma} \mathrm{e}^{(|k|+n) \epsilon}$

for all $k$ and $\forall n \geq 1$.

Next we present a convergence result for $E\left[P_{k}\right]$ as $k \rightarrow \infty$.

Lemma 3.2. Staring with any $P_{0} \in \wp_{n}, E\left[P_{k}\right]$ converges to a bounded matrix $\Gamma^{*} \in \wp_{n}$, where $P_{k}$ satisfies the discrete-time Riccati equations (11).

Proof. As stated above, we can easily verify that the notions of weakly stabilizable and weakly detectable almost surely, introduced in Bougerol (1995) are satisfied. Then by Theorem 5.1 of Bougerol (1995), we know that there exists a unique stationary process $\left\{\bar{P}_{k}\right\}$, with $E\left[\bar{P}_{k}\right]$ constant $\forall k$. That $E\left[\bar{P}_{k}\right] \equiv \Gamma^{*}$ is bounded follows from equation (9) of Bougerol (1995), by setting e.g. $n=0$ to give a bound on $\bar{P}_{0}{ }^{3}$ Furthermore, Theorem 5.3 of Bougerol (1995) shows that $\left\{P_{k}\right\}$ starting from any initial condition $P_{0}$ is exponentially convergent to the stationary process $\left\{\bar{P}_{k}\right\}$. Hence $E\left[P_{k}\right]$ starting from any $P_{0}$ will also converge to $E\left[\bar{P}_{k}\right]=\Gamma^{*}$ as $k \rightarrow \infty$.

In general, analytically evaluating $E\left[P_{k}\right]$ is difficult. Furthermore, even though $E\left[P_{k}\right]$ will be bounded, it is not clear how one can obtain explicit upper bounds for arbitrary fading distributions. We now provide a result on a sequence of deterministic positive semidefinite matrices that overbounds $E\left[P_{k}\right], \forall k$, and also converges to a limit as $k \rightarrow \infty$. Note that $P_{k+1}$ can be regarded as a function of $P_{k}$ and $H_{k}$, so we can write

$$
\begin{aligned}
E\left[P_{k+1}\right]= & E_{H_{k}}\left[E \left[A P_{k} A^{\prime}-A P_{k} C^{\prime}\left(L_{k}^{-1}+C P_{k} C^{\prime}+\Sigma_{v}\right)^{-1}\right.\right. \\
& \left.\left.\times C P_{k} A^{\prime}+\Sigma_{w} \mid H_{k}\right]\right] .
\end{aligned}
$$

\footnotetext{
3 Boundedness of $E\left[P_{k}\right]$ can also be shown by using Theorem 3.3.
} 
Denote $V_{k}=E\left[P_{k}\right]$. Then, by concavity,

$$
\begin{aligned}
V_{k+1} \leq & E_{H_{k}}\left[A V_{k} A^{\prime}-A V_{k} C^{\prime}\left(L_{k}^{-1}+C V_{k} C^{\prime}+\Sigma_{v}\right)^{-1}\right. \\
& \left.\times C V_{k} A^{\prime}+\Sigma_{w}\right] .
\end{aligned}
$$

We have:

Theorem 3.3. For the state space model (4), let $\left\{Z_{k}\right\}$ be defined by

$$
\begin{aligned}
Z_{k+1}= & E_{H_{k}}\left[A Z_{k} A^{\prime}-A Z_{k} C^{\prime}\left(L_{k}^{-1}+C Z_{k} C^{\prime}+\Sigma_{v}\right)^{-1}\right. \\
& \left.\times C Z_{k} A^{\prime}+\Sigma_{w}\right]
\end{aligned}
$$

where $Z_{0}=E\left[P_{0}\right], L_{k}=H_{k} \Sigma_{n}^{-1} H_{k}$, and the components of the diagonal matrix $H_{k}$ are independent and identically distributed with continuous distributions. Then $E\left[P_{k}\right]=V_{k} \leq Z_{k}$, and $Z_{k} \rightarrow Z^{*}$ as $k \rightarrow \infty$, where $Z^{*}$ is the unique fixed point of the recursion (14). $E\left[P_{k}\right]$ starting from $E\left[P_{0}\right]=V_{0}$ converges to a limiting value $\Gamma^{*}$ such that $\Gamma^{*} \leq Z^{*}$.

See Appendix B for the proof.

The bounding matrix sequence $\left\{Z_{k}\right\}$ mentioned above is still difficult to compute in general due to the difficulty of explicitly evaluating the expectation (with respect to the fading gain matrix $H_{k}$ ) of the nonlinear term in the right hand side of Eq. (14). In the next few sections, we show how one can calculate (where evaluating the above expectation is possible) precise bounds for particular cases of systems (e.g, scalar systems, systems with vector states and scalar measurements, and in the case of multisensor systems-scalar state and scalar measurements) along with given fading distribution(s) for the channel(s) connecting the sensor(s) to the FC.

\subsection{Single sensor: Scalar state and measurement}

In this section, we consider the scalar state and measurement model for a single sensor, given by (5). Specializing the errorcovariance notation $P_{k}$ to $p_{k}$ for the scalar case, it is straightforward to show that $p_{k}$ satisfies the following scalar discrete-time Riccati equation:

$p_{k+1}=\sigma_{w}^{2}+\frac{a^{2} p_{k}\left(h_{k}^{2} \sigma_{v}^{2}+\sigma_{n}^{2}\right)}{h_{k}^{2}\left(p_{k}+\sigma_{v}^{2}\right)+\sigma_{n}^{2}}$.

Denoting $h_{k}^{2}$ by $r$, where the time index $k$ has been removed as $\left\{h_{k}\right\}$ is a sequence of i.i.d. random variables, we have as a special case of (13)

$\gamma_{k+1} \leq \sigma_{w}^{2}+a^{2} E_{r}\left[\frac{\gamma_{k}\left(r \sigma_{v}^{2}+\sigma_{n}^{2}\right)}{r\left(\gamma_{k}+\sigma_{v}^{2}\right)+\sigma_{n}^{2}}\right]$

where $\gamma_{k}=E\left[p_{k}\right]$. In order to establish precise upper bounds on $E\left[p_{k}\right]$ as $k \rightarrow \infty$, we consider two specific fading distributions, namely Rayleigh fading and Nakagami fading.

\subsubsection{Rayleigh fading}

In this case $r$ is exponentially distributed with mean $\frac{1}{\lambda}$ such that $r \sim \lambda \exp (-\lambda r)$. It can then be easily shown that

$$
\begin{aligned}
& E_{r}\left[\frac{\gamma_{k}\left(r \sigma_{v}^{2}+\sigma_{n}^{2}\right)}{r\left(\gamma_{k}+\sigma_{v}^{2}\right)+\sigma_{n}^{2}}\right]=\frac{\gamma_{k} \sigma_{v}^{2}}{\gamma_{k}+\sigma_{v}^{2}} E_{r}\left[\frac{r+\frac{\sigma_{n}^{2}}{\sigma_{v}^{2}}}{r+\frac{\sigma_{n}^{2}}{\gamma_{k}+\sigma_{v}^{2}}}\right] \\
& =\frac{\gamma_{k}}{\gamma_{k}+\sigma_{v}^{2}}\left[\sigma_{v}^{2}+\frac{\lambda \sigma_{n}^{2} \gamma_{k}}{\gamma_{k}+\sigma_{v}^{2}} \mathrm{e}^{m_{k}} E_{1}\left(m_{k}\right)\right]
\end{aligned}
$$

where $m_{k}=\frac{\lambda \sigma_{n}^{2}}{\gamma_{k}+\sigma_{v}^{2}}$ and $\mathrm{e}^{x} E_{1}(x)=\int_{0}^{\infty} \frac{\mathrm{e}^{-u}}{u+x} \mathrm{~d} u$, with $E_{1}(x)$ being the exponential integral $E_{1}(x)=\int_{x}^{\infty} \frac{\mathrm{e}^{-t}}{t} \mathrm{~d} t$. Hence

$\gamma_{k+1} \leq \sigma_{w}^{2}+\frac{a^{2} \gamma_{k}}{\gamma_{k}+\sigma_{v}^{2}}\left[\sigma_{v}^{2}+\frac{\lambda \sigma_{n}^{2} \gamma_{k}}{\gamma_{k}+\sigma_{v}^{2}} \mathrm{e}^{m_{k}} E_{1}\left(m_{k}\right)\right]$.
Using the inequality $\mathrm{e}^{x} E_{1}(x)<\ln \left(1+\frac{1}{x}\right)$, one can then write

$$
\begin{aligned}
\gamma_{k+1} & \leq \sigma_{w}^{2}+\frac{a^{2} \gamma_{k}}{\gamma_{k}+\sigma_{v}^{2}}\left[\sigma_{v}^{2}+\frac{\lambda \sigma_{n}^{2} \gamma_{k}}{\gamma_{k}+\sigma_{v}^{2}} \ln \left(1+\frac{\gamma_{k}+\sigma_{v}^{2}}{\lambda \sigma_{n}^{2}}\right)\right] \\
& \leq \sigma_{w}^{2}+a^{2}\left[\sigma_{v}^{2}+\lambda \sigma_{n}^{2} \ln \left(1+\frac{\gamma_{k}+\sigma_{v}^{2}}{\lambda \sigma_{n}^{2}}\right)\right] .
\end{aligned}
$$

We will now define two new sequences $\left\{s_{k}\right\},\left\{q_{k}\right\}$ such that

$$
\begin{aligned}
s_{k+1}= & \sigma_{w}^{2}+\frac{a^{2} s_{k}}{s_{k}+\sigma_{v}^{2}}\left[\sigma_{v}^{2}+\frac{\lambda \sigma_{n}^{2} s_{k}}{s_{k}+\sigma_{v}^{2}} \exp \left(\frac{\lambda \sigma_{n}^{2}}{s_{k}+\sigma_{v}^{2}}\right)\right. \\
& \left.\times E_{1}\left(\frac{\lambda \sigma_{n}^{2}}{s_{k}+\sigma_{v}^{2}}\right)\right] \\
q_{k+1}= & \sigma_{w}^{2}+a^{2}\left[\sigma_{v}^{2}+\lambda \sigma_{n}^{2} \ln \left(1+\frac{q_{k}+\sigma_{v}^{2}}{\lambda \sigma_{n}^{2}}\right)\right]
\end{aligned}
$$

with $s_{0}=E\left[p_{0}\right], q_{0}=E\left[p_{0}\right]$. It is obvious that $s_{k} \leq q_{k}, \forall k$.

One can now provide bounds on $E\left[p_{k}\right]$ in terms of the limiting values of the above sequences as follows:

Theorem 3.4. The sequences $\left\{s_{k}\right\},\left\{q_{k}\right\}$ defined above by (17), (18) converge to their individual limiting values $s^{*}$ and $q^{*}$, respectively as $k \rightarrow \infty$. It is also true that $E\left[p_{k}\right] \leq s_{k} \leq q_{k}, \forall k . E\left[p_{k}\right]$ starting from $E\left[p_{0}\right]$ converges to a limiting value $\gamma^{*}$ where $\gamma^{*} \leq s^{*} \leq q^{*}$.

See Appendix C for the proof. Although convergence of $\left\{s_{k}\right\}$ follows from Theorem 3.3 as a special case (same applies to Theorem 3.5), we provide an alternative and slightly simpler proof for the scalar case, of which the analysis will also be useful for the multisensor situations later.

The sequence $\left\{s_{k}\right\}$ is obviously a tighter bound than $\left\{q_{k}\right\}$. The reason why we consider both sequences is that it is easier to prove convergence by using the $q_{k}$ iterations. In Fig. 1, the average error covariance computed via simulations by averaging over 50000 randomly generated sample paths of length 100 is plotted against the various bounding sequences derived above.

\subsubsection{Nakagami fading}

It is well known that the Nakagami- $m$ distribution provides a very good model for land-mobile and indoor-mobile multipath propagation (Simon \& Alouini, 2000). In this case, the channel power gain $r$ is distributed according to the probability distribution $p_{R}(r)=\frac{m^{m} r^{m-1} \lambda^{m}}{\Gamma(m)} \exp (-\lambda m r)$, where $m \in\left[\frac{1}{2}, \infty\right)$ is a parameter depicting the severity of fading, $\frac{1}{\lambda}$ is the mean channel power gain and $\Gamma$ (.) denotes the gamma function. Note that Rayleigh fading is a special case of Nakagami fading with $m=1$. As $m$ increases beyond 1 , the severity of fading decreases. Here we consider $m=\frac{1}{2}$ which denotes fading that is more severe than Rayleigh fading. In this case, $r \sim \sqrt{\frac{\lambda}{2 \pi r}} \exp \left(-\frac{\lambda r}{2}\right)$.

Denoting $\gamma_{k}=E\left[p_{k}\right]$ as before, we can show from (16) that

$$
\begin{aligned}
\gamma_{k+1} \leq & \sigma_{w}^{2}+\frac{a^{2} \gamma_{k}}{\gamma_{k}+\sigma_{v}^{2}}\left(\sigma_{v}^{2}+\frac{\sigma_{n}^{2} \gamma_{k}}{\gamma_{k}+\sigma_{v}^{2}} \sqrt{\frac{\lambda \pi\left(\sigma_{v}^{2}+\gamma_{k}\right)}{2 \sigma_{n}^{2}}}\right. \\
& \left.\times \exp \left(u^{2}\right) \operatorname{erfc}(u)\right)
\end{aligned}
$$

where $u=\sqrt{\frac{\sigma_{n}^{2} \lambda}{2\left(\sigma_{v}^{2}+\gamma_{k}\right)}}$ and $\frac{\pi}{2 u} \exp \left(u^{2}\right) \operatorname{erfc}(u)=\int_{0}^{\infty} \frac{\mathrm{e}^{-t^{2}}}{t^{2}+u^{2}} \mathrm{~d} t$. Here $\operatorname{erfc}(x)$ denotes the complementary error function defined as $\frac{2}{\sqrt{\pi}} \int_{x}^{\infty} \exp \left(-t^{2}\right) \mathrm{d} t$. Noting that $\frac{\sqrt{\pi}}{2} \exp \left(u^{2}\right) \operatorname{erfc}(u) \leq$ $\frac{1}{u+\sqrt{u^{2}+\frac{4}{\pi}}}$ (Abramowitz \& Stegun, 1972), one can then easily show that 


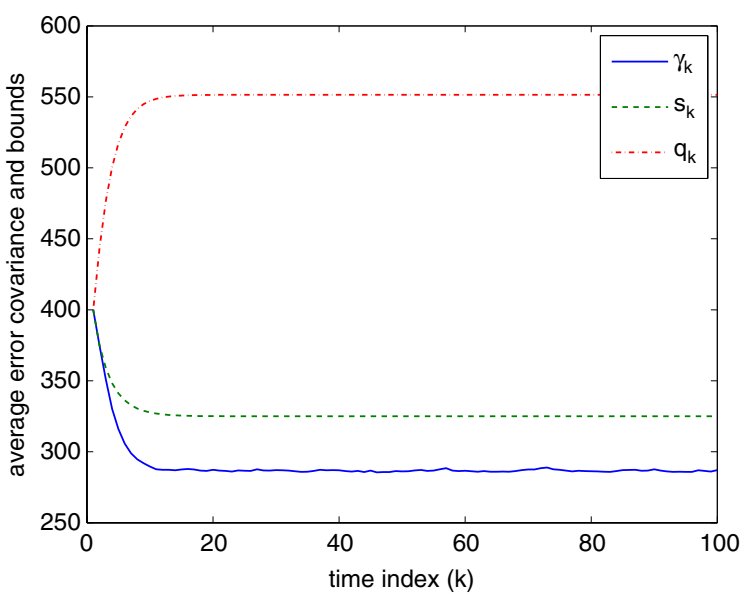

(a) $\sigma_{v}=2.0$.

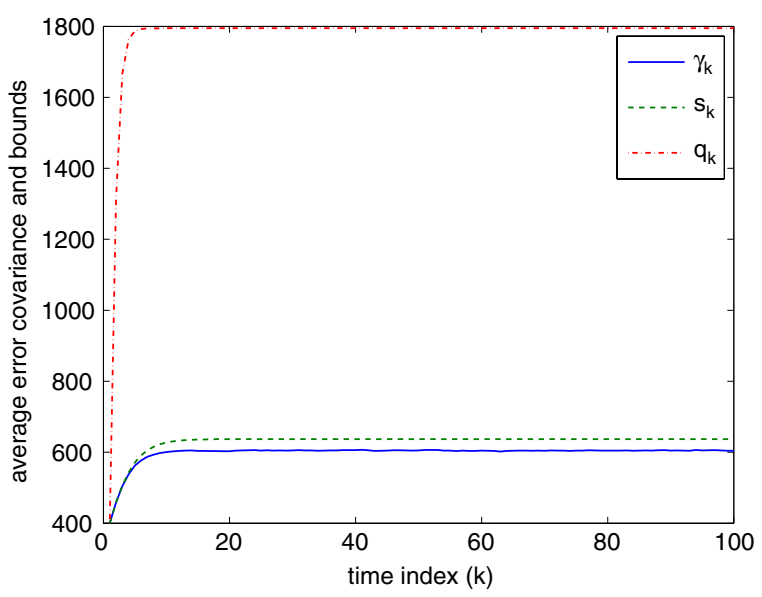

(b) $\sigma_{v}=20.0$.

Fig. 1. Average error covariance and bounds for Rayleigh fading with various $\sigma_{v}$ values, with $a=1.25, c=1.0, \sigma_{w}=1.0, \sigma_{n}=2.0, \lambda=100$.

$\gamma_{k+1} \leq \sigma_{w}^{2}+\frac{a^{2} \gamma_{k}}{\gamma_{k}+\sigma_{v}^{2}}\left(\sigma_{v}^{2}+\frac{\sigma_{n} \sqrt{2 \lambda} \gamma_{k}}{\sqrt{\gamma_{k}+\sigma_{v}^{2}}} \frac{1}{u+\sqrt{u^{2}+\frac{4}{\pi}}}\right)$.

We can now define two sequences $\left\{\tilde{s}_{k}\right\},\left\{\tilde{q}_{k}\right\}$ as follows:

$$
\begin{aligned}
\tilde{s}_{k+1}= & \sigma_{w}^{2}+\frac{a^{2} \tilde{s}_{k}}{\tilde{s}_{k}+\sigma_{v}^{2}}\left(\sigma_{v}^{2}+\frac{\sigma_{n}^{2} \tilde{s}_{k}}{\tilde{s}_{k}+\sigma_{v}^{2}} \sqrt{\frac{\lambda \pi\left(\sigma_{v}^{2}+\tilde{s}_{k}\right)}{2 \sigma_{n}^{2}}}\right. \\
& \left.\times \exp \left(\tilde{u}^{2}\right) \operatorname{erfc}(\tilde{u})\right)
\end{aligned}
$$

$\tilde{q}_{k+1}=\sigma_{w}^{2}+a^{2}\left(\sigma_{v}^{2}+\frac{\sigma_{n} \sqrt{2 \lambda} \tilde{q}_{k}}{\sqrt{\frac{\sigma_{n}^{2} \lambda}{2}}+\sqrt{\frac{\sigma_{n}^{2} \lambda}{2}+\frac{4}{\pi}\left(\tilde{q}_{k}+\sigma_{v}^{2}\right)}}\right)$

with $\tilde{s}_{0}=E\left[p_{0}\right], \tilde{q}_{0}=E\left[p_{0}\right]$, and $\tilde{u}=\sqrt{\frac{\sigma_{n}^{2} \lambda}{2\left(\sigma_{v}^{2}+\tilde{s}_{k}\right)}}$. We can then similarly have the following theorem which states:

Theorem 3.5. For the case of $\operatorname{Nakagami}\left(\frac{1}{2}\right)$ fading, the sequences $\left\{\tilde{s}_{k}\right\},\left\{\tilde{q}_{k}\right\}$ defined above by (19), (20) converge to their individual limiting values $\tilde{s}^{*}$ and $\tilde{q}^{*}$, respectively as $k \rightarrow \infty$. It is also true that $E\left[p_{k}\right] \leq \tilde{s}_{k} \leq \tilde{q}_{k}, \forall k . E\left[p_{k}\right]$ starting from $E\left[p_{0}\right]$ converges to a limiting value $\tilde{\gamma}^{*}$ where $\tilde{\gamma}^{*} \leq \tilde{s}^{*} \leq \tilde{q}^{*}$.

The proof is similar to that of Theorem 3.4 and is omitted.

Fig. 2 shows the simulated average error covariance for Nakagami $\left(\frac{1}{2}\right)$ fading for the same set of parameter values as in Fig. 1. The interpretation of the graphs is similar to that of Fig. 1. Note that, as expected, the average error covariance performance and the corresponding bounds are generally worse than those for Rayleigh fading.

\subsection{Single sensor: Vector state and scalar measurement}

Considering now the vector state scalar measurement model (6), it is easy to show that the corresponding time-varying discretetime Riccati equation is given by

$P_{k+1}=\Sigma_{w}+A\left[P_{k}-\frac{P_{k} \bar{c}^{\prime} \bar{c} P_{k}}{\bar{c} P_{k} \bar{c}^{\prime}+\sigma_{v}^{2}} \frac{r}{r+\beta_{k}}\right] A^{\prime}$

where $r=h_{k}^{2}$ and $\beta_{k}=\frac{\sigma_{n}^{2}}{\bar{c} P_{k} \bar{c}^{\prime}+\sigma_{v}^{2}}$. Note that $\bar{c} P_{k} \bar{c}^{\prime}+\sigma_{v}^{2}$ is clearly a scalar quantity.
As a special case of (13), one can write (by using $\bar{V}_{k}=E\left[P_{k}\right]$ )

$\bar{V}_{k+1} \leq \Sigma_{w}+A \bar{V}_{k} A^{\prime}-\frac{A \bar{V}_{k} \bar{c}^{\prime} \bar{c} \bar{V}_{k} A^{\prime}}{\bar{c} \bar{V}_{k} \bar{c}^{\prime}+\sigma_{v}^{2}} E_{r}\left[1-\frac{\bar{\beta}_{k}}{r+\bar{\beta}_{k}}\right]$

where $\bar{\beta}_{k}=\frac{\sigma_{n}^{2}}{\bar{c} \bar{V}_{k} \bar{c}^{\prime}+\sigma_{v}^{2}}$. In the case of the channel being Rayleigh faded with mean $\frac{1}{\lambda}$, we can then derive (by evaluating the expectation with respect to the channel power gain on the right hand side of the above inequality) the following upper bounding sequence of positive semidefinite matrices $\left\{\bar{Z}_{k}\right\}$ :

$\bar{Z}_{k+1}=\Sigma_{w}+A \bar{Z}_{k} A^{\prime}-\frac{A \bar{Z}_{k} \bar{c}^{\prime} \bar{c} \bar{Z}_{k} A^{\prime}}{\bar{c} \bar{Z}_{k} \bar{c}^{\prime}+\sigma_{v}^{2}} \times\left[1-\bar{m}_{k} \exp \left(\bar{m}_{k}\right) E_{1}\left(\bar{m}_{k}\right)\right]$

with $\bar{Z}_{0}=V_{0}$ and $\bar{m}_{k}=\frac{\lambda \sigma_{n}^{2}}{\bar{c} \bar{Z}_{k} \bar{c}^{\prime}+\sigma_{v}^{2}}$. Following Theorem 3.3, we can now conclude that $E\left[P_{k}\right]=\bar{V}_{k} \leq \bar{Z}_{k}$, and $\bar{Z}_{k} \rightarrow \bar{Z}^{*}$ as $k \rightarrow \infty$. Also, $E\left[P_{k}\right]$ starting from $E\left[P_{0}\right]=\bar{V}_{0}$ converges to a limiting value $\bar{\Gamma}^{*}$ such that $\bar{\Gamma}^{*} \leq \bar{Z}^{*}$. Note that similar results can be obtained for other types of continuous fading distributions. However explicit bounding sequences for these fading distributions are not provided here to avoid repetition.

\subsection{Multiple sensors}

In this section, we consider the case of multiple sensors where each sensor observes a scalar state process and makes a scalar measurement. Both the multi-access (7) and the orthogonal access (8) schemes will be considered. Although these results can be extended to the vector state and scalar measurement (per sensor) case in a similar manner to the previous section, we do not include such results to maintain simplicity. We only consider the case of Rayleigh fading.

\subsubsection{Multi-access scheme}

Recall the signal model (7). The error covariance $p_{k}$ satisfies the recursion

$$
p_{k+1}=\sigma_{w}^{2}+\frac{a^{2} p_{k}\left(\sum_{i=1}^{M} h_{k, i}^{2} \sigma_{i}^{2}+\sigma_{n}^{2}\right)}{\left(\sum_{i=1}^{M} h_{k, i} c_{i}\right)^{2} p_{k}+\sum_{i=1}^{M} h_{k, i}^{2} \sigma_{i}^{2}+\sigma_{n}^{2}}
$$

where for simplicity we call $\sigma_{i}^{2}=\sigma_{v_{i}}^{2}$.

Denoting $\gamma_{k}=E\left[p_{k}\right]$ and letting $r_{i}=h_{i}^{2}$ (dropping the time subscript $k$ ), it follows similar to before that 


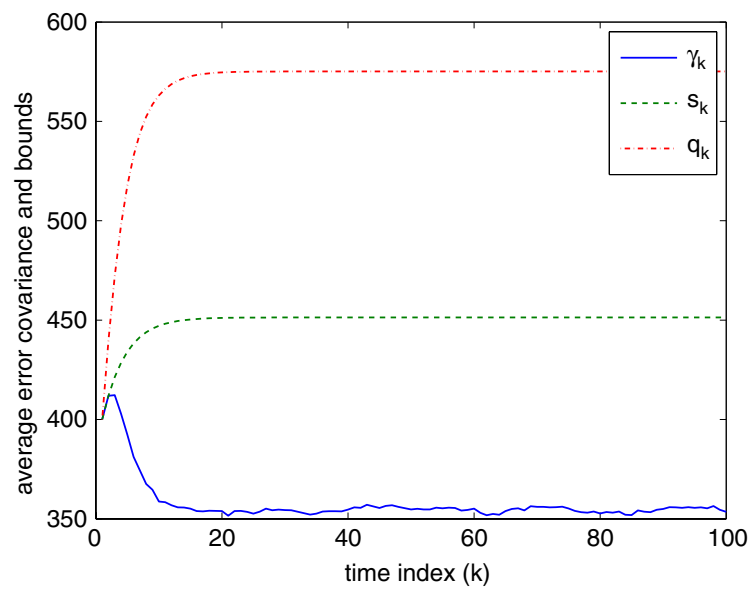

(a) $\sigma_{v}=2.0$.

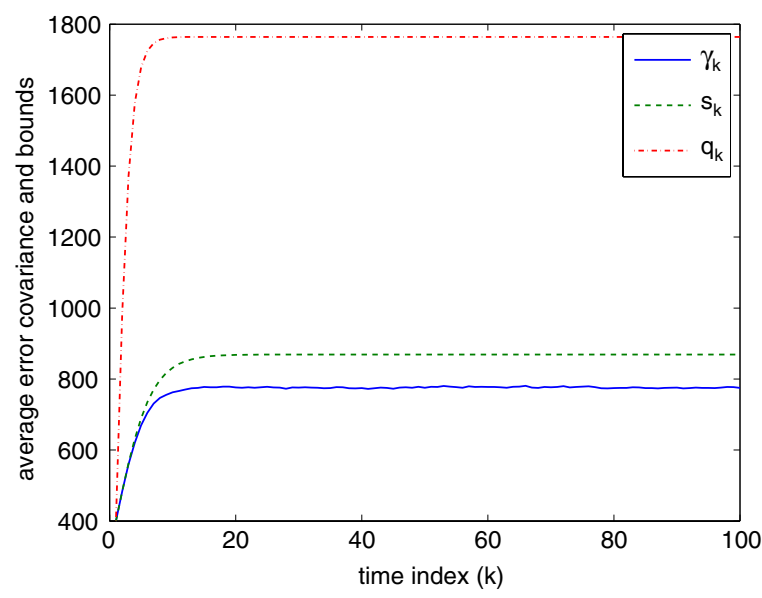

(b) $\sigma_{v}=20.0$.

Fig. 2. Average error covariance and bounds for Nakagami $\left(\frac{1}{2}\right)$ fading with various $\sigma_{v}$ values, with $a=1.25, c=1.0, \sigma_{w}=1.0, \sigma_{n}=2.0, \lambda=100$.

$$
\begin{array}{r}
\gamma_{k+1} \leq \sigma_{w}^{2}+E_{\mathbf{r}}\left[\frac{a^{2} \gamma_{k}\left(\sum_{i=1}^{M} r_{i} \sigma_{i}^{2}+\sigma_{n}^{2}\right)}{\left(\sum_{i=1}^{M} \sqrt{r_{i}} c_{i}\right)^{2} \gamma_{k}+\sum_{i=1}^{M} r_{i} \sigma_{i}^{2}+\sigma_{n}^{2}}\right] \\
\leq \sigma_{w}^{2}+E_{\mathbf{r}}\left[\frac{a^{2} \gamma_{k}\left(\sum_{i=1}^{M} r_{i} \sigma_{i}^{2}+\sigma_{n}^{2}\right)}{\sum_{i=1}^{M} r_{i} c_{i}^{2} \gamma_{k}+\sum_{i=1}^{M} r_{i} \sigma_{i}^{2}+\sigma_{n}^{2}}\right]
\end{array}
$$

where $\mathbf{r}=\left(r_{1}, \ldots, r_{M}\right)$, and the second inequality follows since the $c_{i}$ 's are assumed to be positive. Since we are dealing with Rayleigh fading, $r_{i} \sim \lambda_{i} \exp \left(\lambda_{i} r_{i}\right)$.

Now assume the sensors are ordered in descending order of measurement quality such that

$$
\frac{c_{1}^{2}}{\sigma_{1}^{2}} \geq \frac{c_{2}^{2}}{\sigma_{2}^{2}} \geq \cdots \geq \frac{c_{M}^{2}}{\sigma_{M}^{2}} .
$$

\section{We have}

$$
\begin{aligned}
E_{\mathbf{r}}\left[\frac{\sum_{i=1}^{M} r_{i} \sigma_{i}^{2}+\sigma_{n}^{2}}{\sum_{i=1}^{M} r_{i} c_{i}^{2} \gamma_{k}+\sum_{i=1}^{M} r_{i} \sigma_{i}^{2}+\sigma_{n}^{2}}\right] \\
=\frac{\sigma_{1}^{2}}{c_{1}^{2} \gamma_{k}+\sigma_{1}^{2}} E_{r_{2}, \ldots, r_{M}}\left[1+\left(\mathcal{A}_{1}-\mathscr{B}_{1}\right) \mathrm{e}^{\mathscr{B}_{1}} E_{1}\left(\mathcal{B}_{1}\right)\right]
\end{aligned}
$$

where

$$
\begin{aligned}
& \mathcal{A}_{1}=\frac{\lambda_{1}}{\sigma_{1}^{2}}\left(\sum_{i=2}^{M} r_{i} \sigma_{i}^{2}+\sigma_{n}^{2}\right) \text { and } \\
& \mathcal{B}_{1}=\frac{\lambda_{1}}{c_{1}^{2} \gamma_{k}+\sigma_{1}^{2}}\left(\sum_{i=2}^{M} r_{i}\left(c_{i}^{2} \gamma_{k}+\sigma_{i}^{2}\right)+\sigma_{n}^{2}\right) .
\end{aligned}
$$

By assumption (22), $\mathcal{A}_{1}-\mathscr{B}_{1} \geq 0$. Using the inequality $\mathrm{e}^{x} E_{1}(x)<$ $\ln \left(1+\frac{1}{x}\right)$ would result in very complicated expressions which are difficult to work with for $M>2$. For a simpler expression, we will instead use the looser inequality $\mathrm{e}^{x} E_{1}(x)<\frac{1}{x}$. Then

$$
E_{\mathbf{r}}\left[\frac{\sum_{i=1}^{M} r_{i} \sigma_{i}^{2}+\sigma_{n}^{2}}{\sum_{i=1}^{M} r_{i} c_{i}^{2} \gamma_{k}+\sum_{i=1}^{M} r_{i} \sigma_{i}^{2}+\sigma_{n}^{2}}\right]
$$

$$
\begin{aligned}
& \leq \frac{\sigma_{1}^{2}}{c_{1}^{2} \gamma_{k}+\sigma_{1}^{2}} E_{r_{2}, \ldots, r_{M}}\left[1+\frac{\mathcal{A}_{1}-\mathscr{B}_{1}}{\mathscr{B}_{1}}\right] \\
& =\frac{\sigma_{1}^{2}}{c_{1}^{2} \gamma_{k}+\sigma_{1}^{2}} E_{r_{2}, \ldots, r_{M}}\left[\frac{\mathcal{A}_{1}}{\mathcal{B}_{1}}\right] \\
& =E_{r_{2}, \ldots, r_{M}}\left[\frac{\sum_{i=2}^{M} r_{i} \sigma_{i}^{2}+\sigma_{n}^{2}}{\sum_{2=1}^{M} r_{i}\left(c_{i}^{2} \gamma_{k}+\sigma_{i}^{2}\right)+\sigma_{n}^{2}}\right] \\
& =\frac{\sigma_{2}^{2}}{c_{2}^{2} \gamma_{k}+\sigma_{2}^{2}} E_{r_{3}, \ldots, r_{M}}\left[1+\left(\mathcal{A}_{2}-\mathscr{B}_{2}\right) \mathrm{e}^{\mathscr{B}_{2}} E_{1}\left(\mathscr{B}_{2}\right)\right]
\end{aligned}
$$

where

$$
\begin{aligned}
& \mathcal{A}_{2}=\frac{\lambda_{2}}{\sigma_{2}^{2}}\left(\sum_{i=3}^{M} r_{i} \sigma_{i}^{2}+\sigma_{n}^{2}\right) \text { and } \\
& \mathscr{B}_{2}=\frac{\lambda_{2}}{c_{2}^{2} \gamma_{k}+\sigma_{2}^{2}}\left(\sum_{i=3}^{M} r_{i}\left(c_{i}^{2} \gamma_{k}+\sigma_{i}^{2}\right)+\sigma_{n}^{2}\right) .
\end{aligned}
$$

By assumption (22), we also have $\mathscr{A}_{2}-\mathscr{B}_{2} \geq 0$. Continuing this process, we eventually arrive at

$$
\begin{aligned}
& E_{\mathbf{r}}\left[\frac{\sum_{i=1}^{M} r_{i} \sigma_{i}^{2}+\sigma_{n}^{2}}{\sum_{i=1}^{M} r_{i} c_{i}^{2} \gamma_{k}+\sum_{i=1}^{M} r_{i} \sigma_{i}^{2}+\sigma_{n}^{2}}\right] \leq \frac{\sigma_{M}^{2}}{c_{M}^{2} \gamma_{k}+\sigma_{M}^{2}} \\
& \quad \times\left[1+\left(\mathcal{A}_{M}-\mathcal{B}_{M}\right) \mathrm{e}^{\mathcal{B}_{M}} E_{1}\left(\mathcal{B}_{M}\right)\right]
\end{aligned}
$$

where $\mathcal{A}_{M}=\frac{\lambda_{M}}{\sigma_{M}^{2}} \sigma_{n}^{2}, \mathscr{B}_{M}=\frac{\lambda_{M}}{c_{M}^{2} \gamma_{k}+\sigma_{M}^{2}} \sigma_{n}^{2}$. Hence $\gamma_{k+1} \leq \sigma_{w}^{2}+\frac{a^{2} \gamma_{k} \sigma_{M}^{2}}{c_{M}^{2} \gamma_{k}+\sigma_{M}^{2}}\left[1+\left(\mathcal{A}_{M}-\mathscr{B}_{M}\right) \mathrm{e}^{\mathcal{B}_{M}} E_{1}\left(\mathcal{B}_{M}\right)\right]$.

We can define the bounding sequence

$s_{k+1}=\sigma_{w}^{2}+\frac{a^{2} s_{k} \sigma_{M}^{2}}{c_{M}^{2} s_{k}+\sigma_{M}^{2}}\left[1+\left(\tilde{\mathscr{A}}_{M}-\tilde{\mathscr{B}}_{M}\right) \mathrm{e}^{\tilde{\mathcal{B}}_{M}} E_{1}\left(\tilde{\mathscr{B}}_{M}\right)\right]$

with $\tilde{\mathscr{A}}_{M}=\frac{\lambda_{M}}{\sigma_{M}^{2}} \sigma_{n}^{2}, \tilde{\mathscr{B}}_{M}=\frac{\lambda_{M}}{c_{M}^{2} s_{k}+\sigma_{M}^{2}} \sigma_{n}^{2}$, and convergence properties of this sequence can be proved similar to Theorem 3.4. Recalling the ordering (22), we thus see that we are bounded by the result assuming just the "worst" sensor in terms of the sensor SNR $c_{i}^{2} / \sigma_{i}^{2}$. 


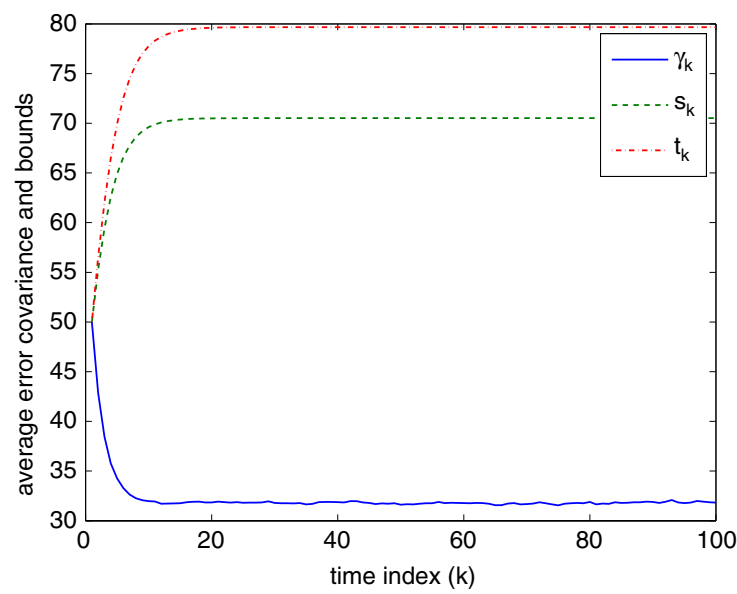

(a) $\lambda_{1}=100, \lambda_{2}=20$.

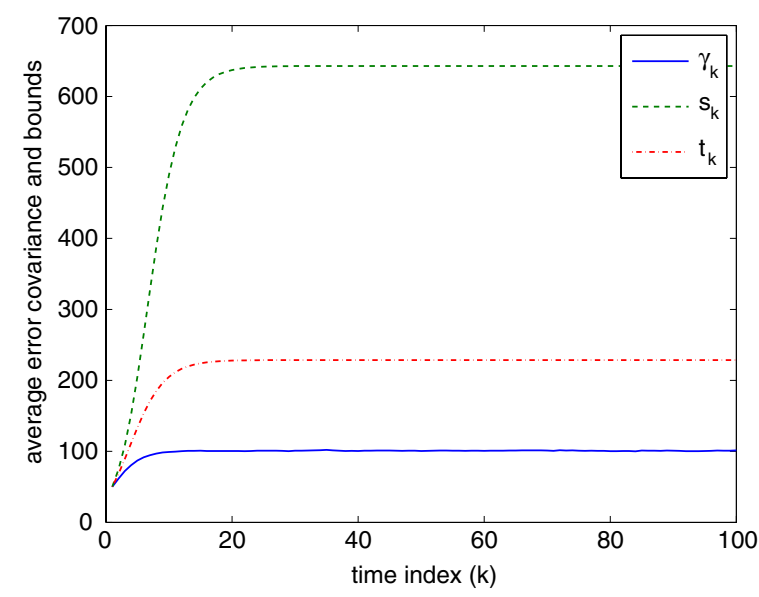

(b) $\lambda_{1}=100, \lambda_{2}=200$.

Fig. 3. Average error covariance and bounds for multi-access scheme, with $a=1.25, c_{1}=1.0, c_{2}=1.0, \sigma_{w}=1.0, \sigma_{1}=1.0, \sigma_{2}=2.0, \sigma_{n}=2.0$.

\section{An alternative bound}

Consider the following inequality for $x_{i} \geq 0$,

$\frac{1}{\sum_{i=1}^{M} x_{i}} \leq \frac{1}{M^{2}} \sum_{i=1}^{M} \frac{1}{x_{i}}$

which is a consequence of the well-known result that the arithmetic mean is greater than or equal to the harmonic mean. We will use this inequality to derive an alternative bound. A more attractive feature of this bound is that the parameters for all of the sensors will appear in the expressions obtained. Applying the inequality (23), we have

$$
\begin{aligned}
& E_{\mathbf{r}}\left[\frac{\sum_{i=1}^{M} r_{i} \sigma_{i}^{2}+\sigma_{n}^{2}}{\sum_{i=1}^{M} r_{i}\left(c_{i}^{2} \gamma_{k}+\sigma_{i}^{2}\right)+\sigma_{n}^{2}}\right]=E_{\mathbf{r}}\left[\frac{\sum_{i=1}^{M} r_{i} \sigma_{i}^{2}+\sigma_{n}^{2}}{\sum_{i=1}^{M}\left(r_{i}\left(c_{i}^{2} \gamma_{k}+\sigma_{i}^{2}\right)+\frac{\sigma_{n}^{2}}{M}\right)}\right] \\
& \leq \frac{1}{M^{2}} E_{\mathbf{r}}\left[\sum_{i=1}^{M} \frac{\sum_{j=1}^{M} r_{j} \sigma_{j}^{2}+\sigma_{n}^{2}}{r_{i}\left(c_{i}^{2} \gamma_{k}+\sigma_{i}^{2}\right)+\frac{\sigma_{n}^{2}}{M}}\right] .
\end{aligned}
$$

\section{We can evaluate}

$$
\begin{gathered}
E_{\mathbf{r}}\left[\frac{\sum_{j=1}^{M} r_{j} \sigma_{j}^{2}+\sigma_{n}^{2}}{r_{i}\left(c_{i}^{2} \gamma_{k}+\sigma_{i}^{2}\right)+\frac{\sigma_{n}^{2}}{M}}\right]=E_{r_{i}}\left[\frac{r_{i} \sigma_{i}^{2}+\sum_{j \neq i} \frac{\sigma_{j}^{2}}{\lambda_{j}}+\sigma_{n}^{2}}{r_{i}\left(c_{i}^{2} \gamma_{k}+\sigma_{i}^{2}\right)+\frac{\sigma_{n}^{2}}{M}}\right] \\
=\frac{\sigma_{i}^{2}}{c_{i}^{2} \gamma_{k}+\sigma_{i}^{2}}\left[1+\left(\mathcal{C}_{i}-\mathscr{D}_{i}\right) \mathrm{e}^{D_{i}} E_{1}\left(D_{i}\right)\right]
\end{gathered}
$$

with $\mathcal{C}_{i}=\frac{\lambda_{i}}{\sigma_{i}^{2}}\left(\sum_{j \neq i} \frac{\sigma_{j}^{2}}{\lambda_{j}}+\sigma_{n}^{2}\right), \mathscr{D}_{i}=\frac{\lambda_{i} \sigma_{n}^{2}}{M\left(c_{i}^{2} \gamma_{k}+\sigma_{i}^{2}\right)}$. Hence an alternative bounding sequence is

$t_{k+1}=\sigma_{w}^{2}+\frac{a^{2} t_{k}}{M^{2}} \sum_{i=1}^{M} \frac{\sigma_{i}^{2}}{c_{i}^{2} t_{k}+\sigma_{i}^{2}}\left[1+\left(\tilde{\mathcal{C}}_{i}-\tilde{D}_{i}\right) \mathrm{e}^{\tilde{D}_{i}} E_{1}\left(\tilde{\mathscr{D}}_{i}\right)\right]$

where $\tilde{\mathcal{C}}_{i}=\frac{\lambda_{i}}{\sigma_{i}^{2}}\left(\sum_{j \neq i} \frac{\sigma_{j}^{2}}{\lambda_{j}}+\sigma_{n}^{2}\right), \tilde{D}_{i}=\frac{\lambda_{i} \sigma_{n}^{2}}{M\left(c_{i}^{2} t_{k}+\sigma_{i}^{2}\right)}$.

\subsubsection{Orthogonal access scheme}

Recalling the orthogonal access model (8), it can be shown using the matrix inversion lemma that the error covariance satisfies

$$
p_{k+1}=\sigma_{w}^{2}+\frac{a^{2} p_{k}}{1+p_{k} \sum_{i=1}^{M} \frac{h_{k, i}^{2} c_{i}^{2}}{h_{k, i}^{2} \sigma_{i}^{2}+\sigma_{n}^{2}}} .
$$

Letting $\gamma_{k}=E\left[p_{k}\right], r_{i}=h_{i}^{2}$, we have

$\gamma_{k+1} \leq \sigma_{w}^{2}+E_{\mathbf{r}}\left[\frac{a^{2} \gamma_{k}}{1+\gamma_{k} \sum_{i=1}^{M} \frac{r_{i} c_{i}^{2}}{r_{i} \sigma_{i}^{2}+\sigma_{n}^{2}}}\right]$

We can compute

$$
\begin{aligned}
& E_{\mathbf{r}}\left[\frac{1}{1+\gamma_{k} \sum_{i=1}^{M} \frac{r_{i} c_{i}^{2}}{r_{i} \sigma_{i}^{2}+\sigma_{n}^{2}}}\right] \\
&=E_{\mathbf{r}}\left[\frac{r_{1} \sigma_{1}^{2}+\sigma_{n}^{2}}{\left(r_{1} \sigma_{1}^{2}+\sigma_{n}^{2}\right)\left(1+\gamma_{k} \sum_{i=2}^{M} \frac{r_{i} c_{i}^{2}}{r_{i} \sigma_{i}^{2}+\sigma_{n}^{2}}\right)+r_{1} c_{1}^{2} \gamma_{k}}\right] \\
&=\frac{\sigma_{1}^{2}}{\sigma_{1}^{2}\left(1+\gamma_{k} \sum_{i=2}^{M} \frac{r_{i} c_{i}^{2}}{r_{i} \sigma_{i}^{2}+\sigma_{n}^{2}}\right)+c_{1}^{2} \gamma_{k}} \\
& \times E_{r_{2}, \ldots, r_{M}\left[1+\left(\mathcal{A}_{1}-\mathcal{B}_{1}\right) \mathrm{e}^{\mathcal{B}_{1}} E_{1}\left(\mathcal{B}_{1}\right)\right]}
\end{aligned}
$$

where

$\mathcal{A}_{1}=\frac{\lambda_{1} \sigma_{n}^{2}}{\sigma_{1}^{2}}, \quad \mathcal{B}_{1}=\frac{\lambda_{1} \sigma_{n}^{2}\left(1+\gamma_{k} \sum_{i=2}^{M} \frac{r_{i} c_{i}^{2}}{r_{i} \sigma_{i}^{2}+\sigma_{n}^{2}}\right)}{\sigma_{1}^{2}\left(1+\gamma_{k} \sum_{i=2}^{M} \frac{r_{i} c_{i}^{2}}{r_{i} \sigma_{i}^{2}+\sigma_{n}^{2}}\right)+c_{1}^{2} \gamma_{k}}$.

We note that here

$$
\mathcal{A}_{1}-\mathscr{B}_{1}=\frac{\lambda_{1} \sigma_{n}^{2} c_{1}^{2} \gamma_{k}}{\sigma_{1}^{2}\left[\sigma_{1}^{2}\left(1+\gamma_{k} \sum_{i=2}^{M} \frac{r_{i} c_{i}^{2}}{r_{i} \sigma_{i}^{2}+\sigma_{n}^{2}}\right)+c_{1}^{2} \gamma_{k}\right]}
$$


is always positive, unlike the multi-access scheme where we needed the extra assumption (22). Again using the inequality $\mathrm{e}^{x} E_{1}(x)<\frac{1}{x}$, we obtain

$$
\begin{aligned}
& E_{\mathbf{r}}\left[\frac{1}{1+\gamma_{k} \sum_{i=1}^{M} \frac{r_{i} c_{i}^{2}}{r_{i} \sigma_{i}^{2}+\sigma_{n}^{2}}}\right] \leq E_{r_{2}, \ldots, r_{M}}\left[\frac{1}{1+\gamma_{k} \sum_{i=2}^{M} \frac{r_{i} c_{i}^{2}}{r_{i} \sigma_{i}^{2}+\sigma_{n}^{2}}}\right] \\
& \quad \leq \cdots \leq \frac{\sigma_{M}^{2}}{c_{M}^{2} \gamma_{k}+\sigma_{M}^{2}}\left[1+\left(\mathcal{A}_{M}-\mathscr{B}_{M}\right) \mathrm{e}^{\mathcal{B}_{M}} E_{1}\left(\mathcal{B}_{M}\right)\right]
\end{aligned}
$$

where $\mathcal{A}_{M}=\frac{\lambda_{M} \sigma_{n}^{2}}{\sigma_{M}^{2}}, \mathcal{B}_{M}=\frac{\lambda_{M} \sigma_{n}^{2}}{\sigma_{M}^{2}+c_{M}^{2} \gamma_{k}}$. Note, however, that since no ordering of the sensors is assumed, we could have taken the expectations over $\left(r_{1}, \ldots, r_{M}\right)$ in any order. Hence we have

$\gamma_{k+1} \leq \sigma_{w}^{2}+\min _{i=1, \ldots, M} \frac{a^{2} \gamma_{k} \sigma_{i}^{2}}{c_{i}^{2} \gamma_{k}+\sigma_{i}^{2}}\left[1+\left(\mathcal{A}_{i}^{\prime}-\mathcal{B}_{i}^{\prime}\right) \mathrm{e}^{\mathcal{B}_{i}^{\prime}} E_{1}\left(\mathcal{B}_{i}^{\prime}\right)\right]$

where $\mathcal{A}_{i}^{\prime}=\frac{\lambda_{i} \sigma_{n}^{2}}{\sigma_{i}^{2}}, \mathcal{B}_{i}^{\prime}=\frac{\lambda_{i} \sigma_{n}^{2}}{\sigma_{i}^{2}+c_{i}^{2} \gamma_{k}}$. We can thus define an upper bounding sequence

$s_{k+1}=\sigma_{w}^{2}+\min _{i=1, \ldots, M} \frac{a^{2} s_{k} \sigma_{i}^{2}}{c_{i}^{2} s_{k}+\sigma_{i}^{2}}\left[1+\left(\tilde{\mathscr{A}}_{i}-\tilde{\mathscr{B}}_{i}\right) \mathrm{e}^{\tilde{\mathscr{B}}_{i}} E_{1}\left(\tilde{\mathscr{B}}_{i}\right)\right]$

with $\tilde{\mathcal{A}}_{i}=\frac{\lambda_{i} \sigma_{n}^{2}}{\sigma_{i}^{2}}, \tilde{\mathcal{B}}_{i}=\frac{\lambda_{i} \sigma_{n}^{2}}{\sigma_{i}^{2}+c_{i}^{2} s_{k}}$. Convergence properties of the sequence can be proved similar to before.

An alternative bound

Similar to the multi-access case, we can derive an alternative bound, again using the inequality (23). For the orthogonal scheme, we get

$$
\begin{gathered}
E_{\mathbf{r}}\left[\frac{1}{1+\gamma_{k} \sum_{i=1}^{M} \frac{r_{i} c_{i}^{2}}{r_{i} \sigma_{i}^{2}+\sigma_{n}^{2}}}\right]=E_{\mathbf{r}}\left[\frac{1}{\sum_{i=1}^{M}\left(\frac{1}{M}+\frac{\gamma_{k} r_{i} c_{i}^{2}}{r_{i} \sigma_{i}^{2}+\sigma_{n}^{2}}\right)}\right] \\
\leq \frac{1}{M^{2}} E_{\mathbf{r}}\left[\sum_{i=1}^{M} \frac{1}{\frac{1}{M}+\frac{\gamma_{k} r_{i} c_{i}^{2}}{r_{i} \sigma_{i}^{2}+\sigma_{n}^{2}}}\right] \\
=\frac{1}{M^{2}} \sum_{i=1}^{M} \frac{M \sigma_{i}^{2}}{M c_{i}^{2} \gamma_{k}+\sigma_{i}^{2}}\left[1+\left(\mathcal{C}_{i}-\mathscr{D}_{i}\right) \mathrm{e}^{D_{i}} E_{1}\left(D_{i}\right)\right]
\end{gathered}
$$

where $\mathcal{C}_{i}=\frac{\lambda_{i} \sigma_{n}^{2}}{\sigma_{i}^{2}}, \mathscr{D}_{i}=\frac{\lambda_{i} \sigma_{n}^{2}}{M c_{i}^{2} \gamma_{k}+\sigma_{i}^{2}}$. Thus an alternative bounding sequence is

$t_{k+1}=\sigma_{w}^{2}+\frac{a^{2} t_{k}}{M^{2}} \sum_{i=1}^{M} \frac{M \sigma_{i}^{2}\left[1+\left(\tilde{\mathcal{C}}_{i}-\tilde{D}_{i}\right) \mathrm{e}^{\tilde{D}_{i}} E_{1}\left(\tilde{D}_{i}\right)\right]}{M c_{i}^{2} t_{k}+\sigma_{i}^{2}}$

where $\tilde{\mathcal{C}}_{i}=\frac{\lambda_{i} \sigma_{n}^{2}}{\sigma_{i}^{2}}, \tilde{D}_{i}=\frac{\lambda_{i} \sigma_{n}^{2}}{M c_{i}^{2} t_{k}+\sigma_{i}^{2}}$.

Note also that the expectation in the second line of (25) can be evaluated without requiring the independence of the channel fading between sensors, so even for spatially correlated channel gains the bound (26) is still valid.

\subsubsection{Simulation results}

In Fig. 3 we plot the simulated average error covariance for the multi-access scheme with two sensors, for different values of $\lambda_{1}$ and $\lambda_{2}$. We also plot, on the same figure, the two bounds $s_{k}$ and $t_{k}$. We see that sometimes both $s_{k}$ will be better than $t_{k}$, but sometimes the alternative bound $t_{k}$ will be better. In Fig. 4 we similarly plot the simulated average error covariance and the two different bounds $s_{k}$ and $t_{k}$, for the orthogonal access scheme with two sensors. Similar interpretations to Fig. 3 apply.

\section{Conclusions and future work}

In this paper, we considered a linear state estimation problem when measurements from single or multiple sensors are received via random fading channels at a remote fusion center. Under some mild assumptions, we showed that the expected (with respect to the fading process) estimation error covariance at the fusion center remains bounded and converges to a steady state value. While explicit expressions of the expected error covariance are hard to compute exactly, we provided exact deterministic bounding sequences on the average error covariance for the system models with scalar measurements (per sensor) and specific fading distributions. Numerical illustrations show that these bounds can often be quite tight. Future research will focus on extension of these results to correlated fading channels (both temporal and spatial correlation), characterizing the asymptotic steady state distribution of the error covariance matrix, and optimal resource allocation problems in wireless sensor networks carrying out linear state estimation under resource and communication constraints using the bounds derived here.

\section{Appendix A. Proof of Lemma 3.1}

Suppose we have a positive semidefinite matrix $Q_{k}$ independent of $H_{k}$ such that $P_{k} \geq Q_{k}$. Note that since $H_{k}$ is independent of $P_{k}$, one can write

$$
\begin{aligned}
G\left(P_{k}\right)= & E_{H_{k}}\left[\operatorname { m i n } _ { K _ { k } } \left\{\left(A-K_{k} C\right) P_{k}\left(A-K_{k} C\right)^{\prime}+\Sigma_{w}\right.\right. \\
& \left.\left.+K_{k}\left(\Sigma_{v}+L_{k}^{-1}\right) K_{k}^{\prime}\right\}\right] \\
= & E_{H_{k}}\left[\left\{\left(A-K_{k}^{*} C\right) P_{k}\left(A-K_{k}^{*} C\right)^{\prime}+\Sigma_{w}\right.\right. \\
& \left.\left.+K_{k}^{*}\left(\Sigma_{v}+L_{k}^{-1}\right) K_{k}^{* \prime}\right\}\right] \\
\geq & E_{H_{k}}\left[\left\{\left(A-K_{k}^{*} C\right) Q_{k}\left(A-K_{k}^{*} C\right)^{\prime}+\Sigma_{w}\right.\right. \\
& \left.\left.+K_{k}^{*}\left(\Sigma_{v}+L_{k}^{-1}\right) K_{k}^{* \prime}\right\}\right] \\
\geq & E_{H_{k}}\left[\operatorname { m i n } _ { K _ { k } } \left\{\left(A-K_{k} C\right) Q_{k}\left(A-K_{k} C\right)^{\prime}+\Sigma_{w}\right.\right. \\
& \left.\left.+K_{k}\left(\Sigma_{v}+L_{k}^{-1}\right) K_{k}^{\prime}\right\}\right] \\
= & G\left(Q_{k}\right)
\end{aligned}
$$

where the first line follows from the fact that the Kalman filter operates with the optimal time-varying gain $K_{k}^{*}$, and the third line follows since $P_{k} \geq Q_{k}$. This completes the proof of the nondecreasing property.

In order to prove that $G\left(P_{k}\right)$ is a concave function of $P_{k}$, one needs to show that

$G\left(\alpha P_{k}^{1}+(1-\alpha) P_{k}^{2}\right) \geq \alpha G\left(P_{k}^{1}\right)+(1-\alpha) G\left(P_{k}^{2}\right)$

where $P_{k}^{1}, P_{k}^{2}$ are both positive semidefinite and $0<\alpha<1$. Suppose $\bar{P}_{k}=\alpha P_{k}^{1}+(1-\alpha) P_{k}^{2}$. Then, using the fact that the Kalman filter operates with the optimal gain, we have

$$
\begin{aligned}
G\left(\bar{P}_{k}\right)= & E_{H_{k}}\left[\operatorname { m i n } _ { X } \left\{(A-X C)\left(\alpha P_{k}^{1}+(1-\alpha) P_{k}^{2}\right)(A-X C)^{\prime}\right.\right. \\
& \left.\left.+\Sigma_{w}+X\left(\Sigma_{v}+L_{k}^{-1}\right) X^{\prime}\right\}\right] \\
= & E_{H_{k}}\left[\min _{X}\left(\alpha f\left(X, P_{k}^{1}\right)+(1-\alpha) f\left(X, P_{k}^{2}\right)\right)\right]
\end{aligned}
$$

where $f\left(X, P_{k}\right)=(A-X C) P_{k}(A-X C)^{\prime}+\Sigma_{w}+X\left(\Sigma_{v}+L_{k}^{-1}\right) X^{\prime}$. Noting 


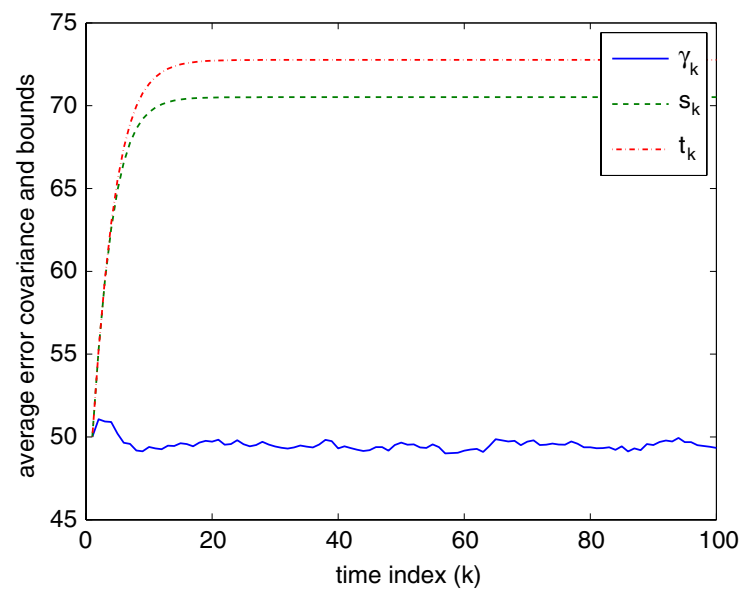

(a) $\lambda_{1}=100, \lambda_{2}=20$.

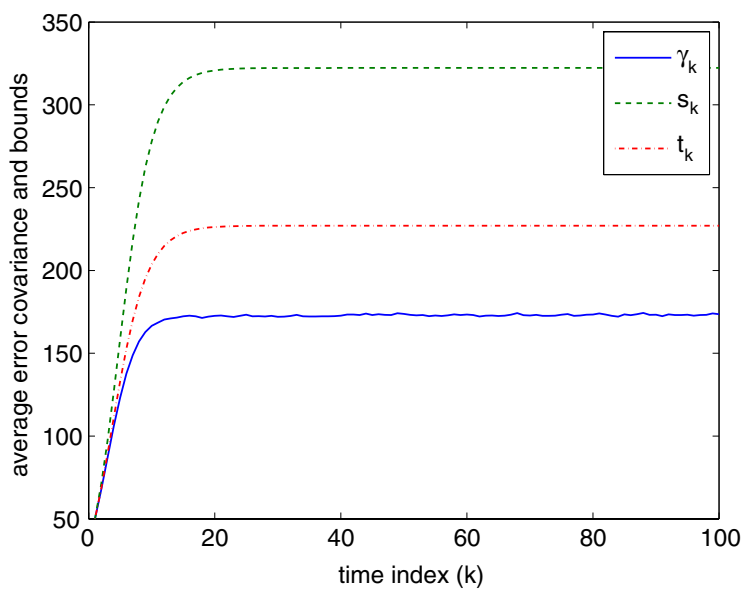

(b) $\lambda_{1}=100, \lambda_{2}=200$.

Fig. 4. Average error covariance and bounds for orthogonal scheme, with $a=1.25, c_{1}=1.0, c_{2}=1.0, \sigma_{w}=1.0, \sigma_{1}=1.0, \sigma_{2}=2.0, \sigma_{n}=2.0$.

that $f\left(X, P_{k}\right)$ is an affine function in $P_{k}$, and pointwise minimum of an affine function is a concave function, it is clear that

$$
\begin{aligned}
G\left(\bar{P}_{k}\right) & \geq E_{h_{k}}\left[\alpha \min _{X} f\left(X, P_{k}^{1}\right)+(1-\alpha) \min _{X} f\left(X, P_{k}^{2}\right)\right] \\
& =\alpha G\left(P_{k}^{1}\right)+(1-\alpha) G\left(P_{k}^{2}\right)
\end{aligned}
$$

which completes the proof of concavity.

\section{Appendix B. Proof of Theorem 3.3}

The fact that $V_{k} \leq Z_{k}$ follows from the non-decreasing property of Lemma 3.1 and induction.

Let us call

$f_{H}(X)=A X A^{\prime}-A X C^{\prime}\left(L^{-1}+C X C^{\prime}+\Sigma_{v}\right)^{-1} C X A^{\prime}+\Sigma_{w}$

where $L=H \Sigma_{n}^{-1} H$. First we show that there exists a fixed point $Z^{*}$ that satisfies

$Z^{*}=E_{H}\left[f_{H}\left(Z^{*}\right)\right]$.

The function $f_{H}(X)$ has the following properties:

(1) $H_{1} \leq H_{2} \Rightarrow f_{H_{1}}(X) \geq f_{H_{2}}(X)^{4}$

(2) $X \leq Y \Rightarrow f_{H}(X) \leq f_{H}(Y)$.

Property (1) can be easily shown by using Corollary 7.7.4 (a) of Horn and Johnson (1985) and Property (2) is well-known.

Define $T: \ell_{n} \rightarrow \ell_{n}$ by

$T(X)=E_{H}\left[f_{H}(X)\right]$.

Then by Theorem 7.A of Zeidler (1986), a fixed point (i.e. a solution to the equation $T(X)=X$ ) will exist if the following five conditions hold:

(i) $T$ is monotone increasing, i.e. $X \leq Y \Rightarrow T(X) \leq T(Y)$,

(ii) $T$ is a compact operator,

(iii) $s_{n}$ is a normal order cone, i.e. $\exists c>0$ and some norm $\|\cdot\|$ such that $0 \leq X \leq Y \Rightarrow\|X\| \leq c\|Y\|, \forall X, Y \in \varsigma_{n}$,

(iv) $\exists u$ with $u \leq T u$,

(v) $\exists v$ with $v \geq T v$.

\footnotetext{
4 For diagonal matrices, $H_{1} \leq H_{2}$ is equivalent to all the diagonal entries of $H_{1}$ being less than or equal to the corresponding diagonal entries of $\mathrm{H}_{2}$.
}

Condition (i) follows from property (2) of the function $f_{H}(X)$. For condition (ii), since $s_{n}$ is a subset of a finite dimensional space, $T$ is continuous, and the semi-definite cone $s_{n}$ is a closed set, compactness of $T$ follows by example 2.10 of Zeidler (1986). For condition (iii), note that $X \leq Y$ implies $\lambda_{\max }(X) \leq \lambda_{\max }(Y)$, where $\lambda_{\max }(X)$ is the largest (positive) eigenvalue of $X$. Since $X$ and $Y$ are symmetric, the singular values are the same as the eigenvalues, hence $\|X\| \leq\|Y\|$ in the spectral norm, and the condition for $s_{n}$ to be a normal order cone is satisfied for $c=1$. For condition (iv), $u=0$ suffices, since $T(0)=E_{H}\left[f_{H}(0)\right]=\Sigma_{w} \geq 0$. It remains to show condition (v). Now for any invertible $H$, one can always find an $X$ such that $f_{H}(X)<X$, by using e.g. the construction in the proof of Theorem 1 of Bitmead, Gevers, Petersen, and Kaye (1985). Let $H^{*}=\operatorname{diag}\left(h^{*}, \ldots, h^{*}\right)$, and let $X^{*}$ (dependent on $H^{*}$ ) satisfy $f_{H^{*}}\left(X^{*}\right)<X^{*}$. Let us write

$$
\begin{aligned}
E_{H}\left[f_{H}\left(X^{*}\right)\right]= & E_{H}\left[f_{H}\left(X^{*}\right) \mid H \geq H^{*}\right] P\left(H \geq H^{*}\right) \\
& +E_{H}\left[f_{H}\left(X^{*}\right) \mid H \geq H^{*}\right] P\left(H \nsucceq H^{*}\right) .
\end{aligned}
$$

Then we have $E_{H}\left[f_{H}\left(X^{*}\right) \mid H \geq H^{*}\right]<X^{*}$ by property (1), and $P\left(H \geq H^{*}\right) \rightarrow 1$ as $h^{*} \rightarrow 0$. We also have that the matrix $E_{H}\left[f_{H}\left(X^{*}\right) \mid H \not H^{*}\right]$ is bounded above by $\Sigma_{w}+A X^{*} A$, and $P(H \geq$ $\left.H^{*}\right) \rightarrow 0$ as $h^{*} \rightarrow 0$. Hence for sufficiently small $h^{*}$, there exists an $X^{*}$ such that $E_{H}\left[f_{H}\left(X^{*}\right)\right] \leq X^{*}$, and condition $(\mathrm{v})$ is satisfied by letting $v$ be this $X^{*}$. Thus all the conditions for the existence of a fixed point $Z^{*}$ such that $Z^{*}=E_{H}\left[f_{H}\left(Z^{*}\right)\right]$ are satisfied.

Finally, we show convergence of $Z_{k}$ to $Z^{*}$ starting from arbitrary initial conditions, thus proving uniqueness of the fixed point $Z^{*}$. We will use Theorem 3.3 of Krasnosel'skii, Vainikko, Zabreiko, Rutitskii, and Stetsenko (1972) (see also Theorem 46.1 of Krasnosel'skiī \& Zabreǐko, 1984), which gives conditions for a fixed point to be unique, provided one actually exists. We need to show that $T$ is " $u_{0}$-concave", i.e. there is some $u_{0} \in \ell_{n}$ such that:

(i) For any nonzero $X \in \varsigma_{n}, \exists \alpha(X)>0, \beta(X)>0$ such that $\alpha u_{0} \leq T X \leq \beta u_{0}$.

(ii) For each $X$ such that $\alpha u_{0} \leq X \leq \beta u_{0}$, and each $t \in(0,1)$, $\exists \eta(X, t)>0$ such that

$$
T(t X) \geq(1+\eta) t T(X) \text {. }
$$

Under the assumption that $\Sigma_{w}>0$, we can easily verify that taking $u_{0}=I$ will satisfy these conditions. Since we have previously shown that there does exist a fixed point $Z^{*}$, Theorem 3.3 of Krasnosel'skii et al. (1972) then shows that $Z_{k}$ converges to $Z^{*}$ for arbitrary $Z_{0} \in \$_{n}$. 


\section{Appendix C. Proof of Theorem 3.4}

Consider the Eqs. (17) and (18). Note that the right hand sides of (17) and (18) are increasing functions of $s_{k}$ and $q_{k}$ respectively, which implies that both $\left\{s_{k}\right\}$ and $\left\{q_{k}\right\}$ are monotonic sequences. Given that $\gamma_{0}=E\left[p_{0}\right]=s_{0}=q_{0}$, it clearly follows that $\gamma_{1} \leq$ $s_{1} \leq q_{1}$. Using the increasing property mentioned above, one can then prove by induction that $\gamma_{k} \leq s_{k} \leq q_{k}, \forall k=1,2, \ldots$. We know that as a special case of Lemma 3.2, $\gamma_{k}=E\left[p_{k}\right]$ converges to a $\operatorname{limit}\left(\operatorname{say} \gamma^{*}\right)$ as $k \rightarrow \infty$.

We now show that $q_{k}$ also converges to a limit (denoted by $q^{*}$ ) as $k \rightarrow \infty$. In order to prove this, we rewrite (18) as $q_{k+1}=$ $l\left(q_{k}\right)$, where $l\left(q_{k}\right)$ represents the right hand side of (18). It is straightforward to show that the mapping $q=l(q)$ has a unique fixed point. However, in order to prove the convergence of the recursion $q_{k+1}=l\left(q_{k}\right)$ we use the standard function properties of the function $l(q)$ (Yates, 1995), namely positivity, monotonicity (these two are obvious) and scalability. In order to show scalability, we have to show that $\beta l(q)>l(\beta q)$ for $\beta>1$. This follows because

$$
\begin{aligned}
& \ln (\left.+\frac{\beta q_{k}+\sigma_{v}^{2}}{\lambda \sigma_{n}^{2}}\right)<\ln \left(1+\frac{\beta\left(q_{k}+\sigma_{v}^{2}\right)}{\lambda \sigma_{n}^{2}}\right) \\
&<\beta \ln \left(1+\frac{q_{k}+\sigma_{v}^{2}}{\lambda \sigma_{n}^{2}}\right)
\end{aligned}
$$

where the first inequality follows since $\beta>1$ and the second inequality follows since $\ln (1+\beta x)<\beta \ln (1+x)$ for $x>0, \beta>1$. Since $l(q)$ is a standard function, the recursion $q_{k+1}=l\left(q_{k}\right)$ will converge to the unique fixed point $q^{*}$.

Now $\left\{s_{k}\right\}$ is a monotonic sequence sandwiched between two convergent sequences $\left\{q_{k}\right\}$ and $\left\{\gamma_{k}\right\}$ (the limits of the two sequences are in general different). Hence $\left\{s_{k}\right\}$ can be bounded from both above and below, so converges to a limit $s^{*}$. Since $\gamma_{k} \leq s_{k} \leq$ $q_{k}, \forall k=1,2, \ldots$, we have $\gamma^{*} \leq s^{*} \leq q^{*}$.

\section{References}

Abramowitz, M., \& Stegun, I. A. (1972). Handbook of mathematical functions. New York: Dover Publications Inc.

Anderson, B. D. O., \& Moore, J. B. (1979). Optimal filtering. New Jersey: Prentice Hall. Anderson, B. D. O., \& Moore, J. B. (1981). Detectability and stabilizability of timevarying discrete-time linear systems. SIAM Journal on Control and Optimization, 19(1), 20-32.

Bitmead, R. R., Gevers, M. R., Petersen, I. R., \& Kaye, R. J. (1985). Monotonicity and stabilizability properties of solutions of the Riccati difference equation: Propositions, lemmas, theorems, fallacious conjectures and counterexamples. Systems and Control Letters, 5(5), 309-315.

Bougerol, P. (1993). Kalman filtering with random coefficients and contractions. SIAM Journal on Control and Optimization, 31(4), 942-959.

Bougerol, P. (1995). Almost sure stabilizability and Riccati's equation of linear systems with random parameters. SIAM Journal on Control and Optimization, 33(3), 702-717.

Chan, S. W., Goodwin, G. C., \& Sin, K. S. (1984). Convergence properties of the Riccati difference equation in optimal filtering of nonstabilizable systems. IEEE Transactions on Automatic Control, 29(2), 110-118.

Cui, S., Xiao, J.-J., Goldsmith, A., Luo, Z.-Q., \& Poor, H. V. (2007). Estimation diversity and energy efficiency in distributed sensing. IEEE Transactions on Signal Processing, 55(9), 4683-4695.

Dana, A. F., Gupta, V., Hespanha, J. P., Hassibi, B., \& Murray, R. M. (2007). Estimation over communication networks: Performance bounds and achievability results. In Proc. American control conf. (pp. 3450-3455).

Gastpar, M., \& Vetterli, M. (2003). Source-channel communication in sensor networks. Springer Lecture Notes in Computer Science, 2634, 162-177.

Horn, R. A., \& Johnson, C. R. (1985). Matrix analysis. Cambridge, United Kingdom: Cambridge University Press.

Huang, M., \& Dey, S. (2007). Stability of Kalman filtering with Markovian packet losses. Automatica, 43, 598-607.

Jazwinski, A. H. (1970). Stochastic processes and filtering theory. New York: Academic Press.

Krasnosel'skii, M. A., Vainikko, G. M., Zabreiko, P. P., Rutitskii, Y. B., \& Stetsenko, V. Y. (1972). Approximate solution of operator equations. Groningen, Netherlands: Wolters-Noordhoff Publishing.

Krasnosel'skī̌, M. A., \& Zabreǐko, P. P. (1984). Geometrical methods of nonlinear analysis. Berlin: Springer-Verlag.
Li, W., \& Dai, H. (2007). Distributed detection in wireless sensor networks using a multiple access channel. IEEE Transactions on Signal Processing 55(3), 822-833.

Mostofi, Y., \& Murray, R. M. (2005). On dropping noisy packets in Kalman filtering over a wireless fading channel. Proc. American control conf. (pp. 4596-4600).

Mudumbai, R., Barriac, G., \& Madhow, U. (2007). On the feasibility of distributed beamforming in wireless networks. IEEE Transactions on Wireless Communications, 6(5), 1754-1763.

Rajasekaran, P. K., Satyanarayana, N., \& Srinath, M. D. (1971). Optimum linear estimation of stochastic signals in the presence of multiplicative noise. IEEE Transactions on Aerospace and Electronic Systems, 7(3), 462-468.

Simon, M., \& Alouini, M.-S. (2000). Digital communication over fading channels. New York: John Wiley and Sons.

Sinopoli, B., Schenato, L., Franceschetti, M., Poolla, K., Jordan, M. I., \& Sastry, S. S. (2004). Kalman filtering with intermittent observations. IEEE Transactions on Automatic Control, 49(9), 1453-1464.

Tugnait, J. K. (1981). Stability of optimum linear estimators of stochastic signals in white multiplicative noise. IEEE Transactions on Automatic Control, 26(3), 757-761.

Xie, L., \& Xie, L. (2008). Stability of a random Riccati equation with Markovian binary switching. IEEE Transactions on Automatic Control, 53(7), 1759-1764.

$\mathrm{Xu}, \mathrm{Y} .$, \& Hespanha, J. P. (2005). Estimation under uncontrolled and controlled communications in networked control systems. Proc. IEEE conf. decision and control (pp. 842-847).

Yates, R. D. (1995). A framework for uplink power control in cellular radio systems. IEEE Journal on Selected Areas in Communications, 13(7), 1341-1347.

Yuan, W., \& Guo, L. (1999). On stability of random Riccati equations. Science in China (Series E), 42(2), 136-148.

Zeidler, E. (1986). Nonlinear functional analysis and its applications I: Fixed-point theorems. New York: Springer-Verlag.

Zhu, B., Sinopoli, B., Poolla, K., \& Sastry, S. (2007). Estimation over wireless sensor networks. Proc. American control conf. (pp. 2732-2737)

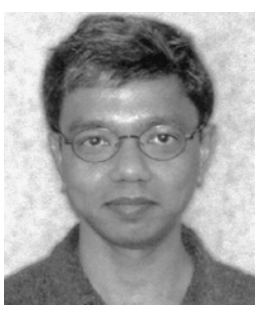

Subhrakanti Dey was born in Calcutta, India, in 1968. He received the B.Tech. and M.Tech. degrees from the Department of Electronics and Electrical Communication Engineering, Indian Institute of Technology, Kharagpur, India, in 1991 and 1993, respectively, and the Ph.D. degree from the Department of Systems Engineering, Research School of Information Sciences and Engineering, Australian National University, Canberra, Australia, in 1996.

He has been with the Department of Electrical and Electronic Engineering, University of Melbourne, Parkville, Australia, since February 2000, where he is currently a full Professor. From September 1995 to September 1997 and September 1998 to February 2000, he was a postdoctoral Research Fellow with the Department of Systems Engineering, Australian National University. From September 1997 to September 1998, he was a post-doctoral Research Associate with the Institute for Systems Research, University of Maryland, College Park. His current research interests include networked control systems, wireless communications and networks, signal processing for sensor networks, and stochastic and adaptive estimation and control.

Dr. Dey currently serves on the Editorial Board of the IEEE Transactions on Signal Processing and Elsevier Systems and Control Letters. He was also an Associate Editor for the IEEE Transactions on Automatic Control during 2005-2007. He is a Senior Member of IEEE.

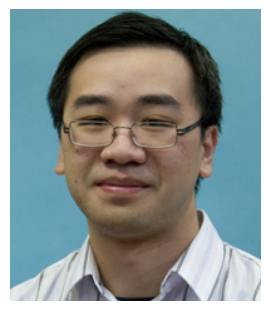

Alex S. Leong was born in Macau in 1980. He received the B.S. degree in mathematics and B.E. degree in Electrical Engineering in 2003, and Ph.D. degree in Electrical Engineering in 2008, all from the University of Melbourne.

He is currently a research fellow in the Department of Electrical and Electronic Engineering at the University of Melbourne. His research interests include statistical signal processing, signal processing for sensor networks, and networked control systems.

Dr. Leong was the recipient of the L. R. East Medal (2003) from Engineers Australia and an Australian Postdoctoral Fellowship (2009) from the Australian Research Council.

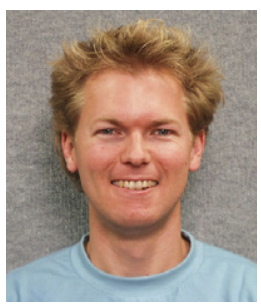

Jamie S. Evans was born in Newcastle, Australia, in 1970 He received the B.S. degree in Physics and the B.E. degree in Computer Engineering from the University of Newcastle, in 1992 and 1993, respectively, where he received the University Medal upon graduation. He received the M.S. and the Ph.D. degrees from the University of Melbourne, Parkville, Australia, in 1996 and 1998, respectively, both in Electrical Engineering, and was awarded the Chancellor's Prize for excellence for his Ph.D. thesis. From March 1998 to June 1999, he was a Visiting Researcher in the Department of Electrical Engineering and Computer Science, University of California, Berkeley. He returned to Australia to take up a position as Lecturer at the University of Sydney, Australia, where he stayed until July 2001. Since that time, he has been with the Department of Electrical and Electronic Engineering, University of Melbourne, where he is now an Associate Professor and Reader. His research interests are in communications theory, information theory, and statistical signal processing with a focus on wireless communications networks. 